\title{
Analytic black branes in Lifshitz-like backgrounds and thermalization
}

\author{
Irina Ya. Aref'eva, ${ }^{a}$ Anastasia A. Golubtsova ${ }^{b, c, d}$ and Eric Gourgoulhon ${ }^{e}$ \\ ${ }^{a}$ Steklov Mathematical Institute, Russian Academy of Sciences, \\ Gubkina str. 8, 119991, Moscow, Russia \\ ${ }^{b}$ Bogoliubov Laboratory of Theoretical Physics, Joint Institute for Nuclear research, \\ Joliot-Curie str. 6, Dubna, 141980, Russia \\ ${ }^{c}$ Dubna State University, \\ Universitetskaya str. 19, Dubna, 141980 , Russia \\ 'Peoples' Friendship University of Russia, \\ Miklukho-Maklaya str. 6, Moscow, 117198, Russia \\ ${ }^{e}$ Laboratoire Univers et Théories, Observatoire de Paris, CNRS, Université Paris Diderot, \\ 5 place Jules Janssen, 92190 Meudon, France \\ E-mail: arefeva@mi.ras.ru, siedhe@gmail.com, eric.gourgoulhon@obspm.fr
}

ABSTRACT: Using black brane solutions in 5d Lifshitz-like backgrounds with arbitrary dynamical exponent $\nu$, we construct the Vaidya geometry, asymptoting to the Lifshitz-like spacetime, which represents a thin shell infalling at the speed of light. We apply the new Lifshitz-Vaidya background to study the thermalization process of the quark-gluon plasma via the thin shell approach previously successfully used in several backgrounds. We find that the thermalization depends on the chosen direction because of the spatial anisotropy. The plasma thermalizes thus faster in the transversal direction than in the longitudinal one. To probe the system described by the Lifshitz-like backgrounds, we also calculate the holographic entanglement entropy for the subsystems delineated along both transversal and longitudinal directions. We show that the entropy has some universality in the behavior for both subsystems. At the same time, we find that certain characteristics strongly depend on the critical exponent $\nu$.

KEYWORDS: Gauge-gravity correspondence, Holography and quark-gluon plasmas, AdSCFT Correspondence

ARXIV EPRINT: 1601.06046 


\section{Contents}

1 Introduction 1

2 Gravity backgrounds 3

2.1 Black branes in Lifshitz-like backgrounds 3

2.2 The Vaidya-Lifshitz geometry 5

3 The thermalization process $\quad 6$

$\begin{array}{lll}3.1 & \text { Thermalization along the longitudinal direction } & 6\end{array}$

$\begin{array}{ll}3.2 & \text { Thermalization along the transversal direction }\end{array}$

4 Entanglement entropy $\quad 9$

4.1 Entanglement entropy in a time-independent background 9

$\begin{array}{llr}\text { 4.1.1 Subsystem delineated along the longitudinal direction } & 9\end{array}$

4.1.2 Subsystem delineated along the transversal direction 11

4.2 Entanglement entropy in a time-dependent background 13

4.2.1 Subsystem delineated along the longitudinal direction 13

$\begin{array}{lll}\text { 4.2.2 Subsystem delineated along the transversal direction } & 16\end{array}$

5 Conclusions $\quad 21$

A Einstein equations $\quad \mathbf{2 5}$

A.1 The l.h.s. of the Einstein equations 25

A.2 The r.h.s. of the Einstein equations 26

B Details on solutions to profiles equations $\quad 27$

B.1 Equations (4.37), (4.38) 27

$\begin{array}{ll}\text { B.2 Equations (4.63), (4.65) } & 29\end{array}$

\section{Introduction}

The gravity/gauge duality provide an alternative tool for understanding dynamics of the strong coupling system, where standard methods lacks. One such system is the quark-gluon plasma (QGP), which can be produced in heavy-ion collisions and represents a strongly coupled fluid with a small viscosity [1]. The QGP goes through several stages of evolution. It is believed that the QGP is created after a very short time after the collision $\tau_{\text {therm }} \approx$ few $0.1 \mathrm{fm} / c$ and the holographic approach, in particular, is aimed to describe this and a short nearest period of evolution $[2,3]$. There are indications that in this time the QGP is anisotropic. Since at time scales of $\tau \approx$ few $0.1 \mathrm{fm} / c$ it is in thermal equilibrium, one can try to apply anisotropic holographic hydrodynamics to describe its isotropization. The 
anisotropic stage of the QGP takes place for $0.1 \mathrm{fm} / c \lesssim \tau \lesssim 0.3-2 \mathrm{fm} / c$ [4] and can be studied also holographically $[5,6]$.

Through the gauge/gravity duality, the thermalization of the field theory in the boundary corresponds to the process of black hole formation in the bulk. According to the holographic dictionary, the scenario of a heavy-ion collision can be represented as a shock wave collision in which trapped surface is formed [7]-[16]. After the collision the shocks slowly decay, leaving the plasma described by hydrodynamics in the middle. The creation of the black hole is also described by the Vaidya metric of an infalling shell with a horizon corresponding to the location of the trapped surface [17]-[21].

By now both standard existing models and the holographic approach with AdS backgrounds, as well as its conformally equivalent deformations for bulk geometries, have failed to reproduce the particle multiplicity at high energies. However, if one performs the holographic estimations of multiplicities in Lifshitz-like spacetimes [22, 23, 25], one can fit the experimental data for certain values of the critical exponents [24]. In this paper, we consider following the 5-dimensional Lifshitz-like metric:

$$
d s^{2}=L^{2}\left[\frac{\left(-d t^{2}+d x^{2}\right)}{z^{2}}+\frac{\left(d y_{1}^{2}+d y_{2}^{2}\right)}{z^{2 / \nu}}+\frac{d z^{2}}{z^{2}}\right] .
$$

The choice of the geometry (1.1) is motivated by studies of the anisotropic phase of the QGP. As it is known, the QGP in the 4d gauge theory can be characterized by the energymomentum tensor $\left\langle T_{\mu \nu}\right\rangle=\operatorname{diag}\left(\varepsilon, p_{L}, p_{T}, p_{T}\right)$, with the particle momenta $\left\langle p_{L}^{2}\right\rangle\left\langle\left\langle p_{T}^{2}\right\rangle\right.$ at early times of the QGP formation. To reproduce this anisotropy from the gravity side, one of the possible backgrounds is the Lifshitz-like metric (1.1). It has been shown in [24] that for the wall-on-wall collision in the $5 \mathrm{~d}$ Lifshitz-like background with the critical exponent $\nu=4$, the dependence of multiplicity on the energy is desirable, i.e. behaves as $E^{1 / 3}$.

Another possible implementation of the $5 \mathrm{~d}$ Lifshitz-like spacetime is

$$
d s^{2}=L^{2}\left[\frac{-d t^{2}}{z^{2}}+\frac{d x^{2}}{z^{2 / \nu}}+\frac{\left(d y_{1}^{2}+d y_{2}^{2}\right)}{z^{2}}+\frac{d z^{2}}{z^{2}}\right],
$$

which differs from (1.1) by the anisotropic scaling taking place only for one spatial direction. The embedding of this background and its non-zero temperature generalization into supergravity IIB was done in [25] for $\nu=3 / 2$. Solutions interpolating between Lifshitz-like (1.2) and AdS geometries were intensively studied in [26]-[32] within the context of applications to the anisotropic QGP. However, the results for multiplicities calculated using the background (1.2) in [24] do not fit the experimental data unlike the case of the metric (1.1).

Since after the shock wave collision the trapped surface argument supports black hole formation, it is natural to construct the corresponding Vaidya-type solution. In the present paper we start from the generalization of (1.1) to the non-zero temperature case for an arbitrary critical exponent. Further, we construct a Vaidya-type geometry asymptoting to the Lifshitz-like solution to model a gravitation collapse in order to study the holographic thermalization. The Vaidya metric with Lifshitz scaling was used for the examination of the holographic thermalization in $[33,34]$. There, it has been shown that for the metric 
with anisotropy between time and spatial directions the propagation of thermalization represents a similar "horizon" behavior as that seen in the AdS case. The Vaidya metric was also generalized to the Lifshitz spacetimes with a hyperscaling violating factor [35-37].

As another application of our solutions, we consider the time evolution of the holographic entanglement entropy during the process of thermalization. The behavior of the entanglement entropy modeling the thermalization and "quench" processes is a subject of intensive studies during last years, see [36, 38-42] and refs. therein. For Lifshitz metrics the time evolution of the entanglement entropy turns out to have a linear regime [33]. In this work, we examine the influence of spatial anisotropy on the behavior of the entanglement entropy.

The paper is organized as follows. Section 2 is devoted to constructing the exact solutions which asymptotes to the Lifshitz-like metric (1.1). In section 2.1 we present the $5 \mathrm{~d}$ black brane background. In section 2.2 we generalize it to the Vaidya type solution, which describes a thin shell collapsing to a black hole in the Lifshitz-like background. In section 3 we numerically calculate thermalization times using our Lifshtiz-Vaidya type solution. Section 4 is devoted to studies of the holographic entanglement entropy at equilibrium as well as its out-of-equilibrium behavior. We conclude in section 5 with a discussion of our results. Appendix A collects some technical details used for constructing analytic solutions. In appendix B we present some details concerning numerical solutions to EOM related the functional of the entanglement entropy.

\section{Gravity backgrounds}

\subsection{Black branes in Lifshitz-like backgrounds}

In [24] we considered a collision of two domain walls in the five-dimensional Lifshitz-like background

$$
d s^{2}=\tilde{r}^{2 \nu}\left(-d t^{2}+d x^{2}\right)+\tilde{r}^{2}\left(d y_{1}^{2}+d y_{2}^{2}\right)+\frac{d \tilde{r}^{2}}{\tilde{r}^{2}},
$$

where $\nu$ is the critical exponent. Note that (2.1) is equivalent to (1.1) via the change of coordinate $z=\tilde{r}^{-\nu}$ and the rescaling $\left(t, x, y_{1}, y_{2}\right) \mapsto \nu^{-1}\left(t, x, y_{1}, y_{2}\right)$.

In $[24,25]$ the metric ansatz $(2.1)$ was considered for a $5 \mathrm{~d}$ model governed by the action

$$
S=\frac{1}{16 \pi G_{5}} \int d^{5} x \sqrt{|g|}\left(R+\Lambda-\frac{1}{6}\left(H_{(3)}^{2}+m_{0}^{2} B_{(2)}^{2}\right)\right),
$$

where $m_{0}$ and $\Lambda$ are constant and the 3 -form $H_{(3)}$ and the 2 -form $B_{(2)}$ are related by

$$
H_{(3)}=d B_{(2)} \text {. }
$$

However, it seems difficult to find an analytic black brane (hole) solution for the model (2.2) due the dependence (2.3) for the gauge fields.

In this paper we consider another bulk theory, possessing the metric (2.1) as a solution to Einstein equations, with the following action

$$
S=\int d^{5} x \sqrt{|g|}\left(R[g]+\Lambda-\frac{1}{2}(\partial \phi)^{2}-\frac{1}{4} e^{\lambda \phi} F_{(2)}^{2}\right),
$$


where the 2 -form $F_{(2)}$ is the gauge field with

$$
F_{(2)}^{2}=F_{m n} F^{m n}
$$

$\phi$ is the dilaton scalar field, $\lambda$ is a dilatonic coupling constant and $\Lambda$ is the cosmological constant. ${ }^{1}$ The model (2.4) can be considered as a truncated supergravity $I I A$ in the style of [25]. Another possible underlying theory is the $5 \mathrm{~d} \mathrm{SO}(6)$ gauged supergravity [44].

The Einstein equations of motion can be written as

$$
R_{m n}=-\frac{\Lambda}{3} g_{m n}+\frac{1}{2}\left(\partial_{m} \phi\right)\left(\partial_{n} \phi\right)+\frac{1}{2} e^{\lambda \phi} F_{m p} F_{n}{ }^{p}-\frac{1}{12} e^{\lambda \phi} F_{(2)}^{2} g_{m n} .
$$

The scalar field equation is

$$
\square \phi=\frac{1}{4} \lambda e^{\lambda \phi} F_{(2)}^{2}, \quad \text { with } \square \phi=\frac{1}{\sqrt{|g|}} \partial_{m}\left(g^{m n} \sqrt{|g|} \partial_{n} \phi\right) .
$$

Finally the equation of motion for the gauge field is

$$
D_{m}\left(e^{\lambda \phi} F^{m n}\right)=0 .
$$

Introducing the new variable

$$
r=\ln \tilde{r},
$$

one can rewrite $(2.1)$ as

$$
d s^{2}=e^{2 \nu r}\left(-d t^{2}+d x^{2}\right)+e^{2 r}\left(d y_{1}^{2}+d y_{2}^{2}\right)+d r^{2} .
$$

We then select the following anzatz for the dilaton and Maxwell fields:

$$
\begin{aligned}
\phi & =\phi(r), \quad e^{\lambda \phi}=\mu e^{4 r}, \\
F_{(2)} & =\frac{1}{2} q d y_{1} \wedge d y_{2},
\end{aligned}
$$

where $\mu$ and $q$ are two constants. One can see that this ansatz has some features. Firstly, the dilaton has the linear dependence in the radial coordinate (2.11). Black hole solutions with a linear dilaton in the supergravity context were discussed in [43]. At the same time the similar ansatz for the gauge fields (2.12) emerges to support $A d S_{2} \times \mathbb{R}^{3}, A d S_{3} \times \mathbb{R}^{2}, A d S_{2} \times$ $\mathbb{R}^{2}$ solutions and their non-zero temperature analogues of gauged supergravity in [44]-[45]. The 6-dimensional Lif $_{4} \times \mathbb{R}^{2}$ background with a constant two-form field was found in [46].

The model (2.4) with the fields given by (2.11)-(2.12) can be generalized to the nonzero temperature case without changing the field ansatz. The metric of the black brane solution reads

$$
d s^{2}=e^{2 \nu r}\left(-f(r) d t^{2}+d x^{2}\right)+e^{2 r}\left(d y_{1}^{2}+d y_{2}^{2}\right)+\frac{d r^{2}}{f(r)},
$$

with the blackening function given by

$$
f(r)=1-m e^{-(2 \nu+2) r} .
$$

\footnotetext{
${ }^{1}$ More precisely, $\Lambda=-2 \bar{\Lambda}$, where $\bar{\Lambda}$ is the standard cosmological constant.
} 
For the particular case $\nu=4$, the metric (2.13)-(2.14) along with the ansatz (2.11)-(2.12) solves the field equations (2.6)-(2.8) provided that the constants take the following values:

$$
\lambda= \pm \frac{2}{\sqrt{3}}, \quad \Lambda=90, \quad \mu q^{2}=240 .
$$

See appendix A.1 for details.

If the dilaton is constant and the Maxwell field vanishes, the metric (2.13) with $\nu=1$ turns out to be the black brane solution in the AdS background:

$$
d s^{2}=e^{2 r}\left(-f(r) d t^{2}+d x^{2}\right)+e^{2 r}\left(d y_{1}^{2}+d y_{2}^{2}\right)+\frac{d r^{2}}{f(r)}
$$

with

$$
f(r)=1-m e^{-4 r}
$$

or in terms of variable $\tilde{r}$

$$
d s^{2}=\tilde{r}^{2}\left(-f(\tilde{r}) d t^{2}+d x^{2}\right)+\tilde{r}^{2}\left(d y_{1}^{2}+d y_{2}^{2}\right)+\frac{d \tilde{r}^{2}}{f(\tilde{r}) \tilde{r}^{2}},
$$

where

$$
f(\tilde{r})=1-\frac{m}{\tilde{r}^{4}}
$$

This corresponds to $r \rightarrow 0$ or the UV limit.

\subsection{The Vaidya-Lifshitz geometry}

To study the thermalization process we need to use the infalling shell approach based on the Vaidya solution [47]. First, we introduce the coordinate $z=e^{-\nu r}$, which, after the rescaling $\left(t, x, y_{1}, y_{2}\right) \mapsto \nu^{-1}\left(t, x, y_{1}, y_{2}\right)$, allows one to rewrite the metric (2.13) in the form

$$
d s^{2}=z^{-2}\left(-f(z) d t^{2}+d x^{2}\right)+z^{-2 / \nu}\left(d y_{1}^{2}+d y_{2}^{2}\right)+\frac{d z^{2}}{z^{2} f(z)},
$$

with the blackening function

$$
f=1-m z^{2+2 / \nu} .
$$

To write down the Vaidya-Lifshitz solution, one should consider the ingoing null geodesics

$$
d t+\frac{d z}{f(z)}=0
$$

and introduce the Eddington-Finkelstein coordinate system $\left(v, x, y_{1}, y_{2}, z\right)$ via

$$
d v=d t+\frac{d z}{f(z)} .
$$

Owing to (2.23) we can represent (2.20) in the following form

$$
d s^{2}=-z^{-2} f(v, z) d v^{2}-2 z^{-2} d v d z+z^{-2} d x^{2}+z^{-2 / \nu}\left(d y_{1}^{2}+d y_{2}^{2}\right),
$$


with

$$
f(v, z)=1-m(v) z^{2+2 / \nu}
$$

where the mass function $m(v)$ determines the thickness of the shell falling along $v=0$ and captures the information about the black hole formation. For the infinite thin shell $m(v)$ has the form

$$
m(v)=M \theta(v),
$$

where $M$ is a constant and $\theta(v)$ is the Heaviside function. One can also consider a smooth function $m(v)$ and get, for instance,

$$
f(v, z)=1-\frac{m}{2}\left(1+\tanh \frac{v}{\alpha}\right) z^{2+2 / \nu}
$$

where $m$ and $\alpha$ are two constants.

The solution (2.24)-(2.25) interpolates between the vacuum Lifshitz solution (2.1) inside the shell $(v<0)$ and the Lifshitz black brane geometry $(2.20)-(2.21)$ outside the shell $(v>0)$.

The check of the equations of motion for the background (2.24)-(2.25) is given in appendix A.2.

\section{The thermalization process}

In [24] we have shown that there is a trapped surface, which forms in the collision of two shock waves in the background (2.1), controlled by boundary points $z_{a}$ and $z_{b}$, with $z_{a}<z_{b}$. This trapped surface defines the location of the horizon for (2.24)-(2.25).

Calculations of the thermalization time $t_{\text {therm }}$ at the scale $\ell$ is based on finding geodesics with endpoints located at the distance $\ell$ for a bulk particle. Then, the thermalization time $t_{\text {therm }}$ is the time when this geodesic covered by the shell (2.24)-(2.25).

The general case for the Lagrangian of the pointlike probe has the form

$$
\mathcal{L}=\sqrt{-z^{-2} f(z) \frac{d v}{d \tau} \frac{d v}{d \tau}-2 z^{-2} \frac{d v}{d \tau} \frac{d z}{d \tau}+z^{-2} \frac{d x}{d \tau} \frac{d x}{d \tau}+z^{-2 / \nu}\left(\frac{d y_{1}}{d \tau} \frac{d y_{1}}{d \tau}+\frac{d y_{2}}{d \tau} \frac{d y_{2}}{d \tau}\right)}
$$

where $\tau$ is a parameter. Here we have two possibilities for the choice of $\tau$ with respect to the transverse directions.

\subsection{Thermalization along the longitudinal direction}

Consider the first case taking $\tau=x$, which can be interpreted as a longitudinal direction. Now we obtain

$$
\mathcal{L}=\frac{\sqrt{\mathcal{R}_{x}}}{z}
$$

where we define

$$
\mathcal{R}=1-f(z)\left(v_{x}^{\prime}\right)^{2}-2 v_{x}^{\prime} z_{x}^{\prime}
$$


The integrals of motion corresponding to (3.2) are

$$
\begin{aligned}
& \mathcal{J}=-\frac{1}{z \sqrt{\mathcal{R}_{x}}}, \\
& \mathcal{I}=\frac{f(z) v_{x}^{\prime}+z_{x}^{\prime}}{z \sqrt{\mathcal{R}}} .
\end{aligned}
$$

From the relations (3.4) and (3.5) we get

$$
z_{x}^{\prime}= \pm \sqrt{f(z)\left(\frac{1}{z^{2} \mathcal{J}^{2}}-1\right)+\frac{\mathcal{I}^{2}}{\mathcal{J}^{2}}}
$$

The turning point $z_{*}$ can be found from the equation

$$
f\left(z_{*}\right)\left(\frac{1}{z_{*}^{2}}-\mathcal{J}^{2}\right)+\mathcal{I}^{2}=0 .
$$

For simplicity, we put $\mathcal{I}=0$ and we get from (3.7)

$$
\mathcal{J}^{2}=\frac{1}{z_{*}^{2}}
$$

For the distance $\ell$ between the ends of the geodesic and the thermalization time one gets

$$
\begin{aligned}
\ell & =2 z_{*} \int_{0}^{1} \frac{w d w}{\sqrt{f\left(z_{*} w\right)\left(1-w^{2}\right)}}, \\
t_{\text {therm }} & =z_{*} \int_{0}^{1} \frac{d w}{f\left(z_{*} w\right)} .
\end{aligned}
$$

Note that here we assume that the turning point lies above the horizon, i.e. $z_{h}>z_{*}$. The behavior of the thermalization time as a function of the distances for (3.9)-(3.10) is represented in figure 1.A. We see that the thermalization time behaves linearly with $\ell$. The results match to those for modified AdS models from [34] and coincide for all values of the dynamical exponent $\nu$.

\subsection{Thermalization along the transversal direction}

Now turn to the second case when $\tau=y_{1}$, that we interpret as the thermalization along a transversal direction. From (3.1) we have

$$
\mathcal{L}=\frac{\sqrt{\mathcal{R}}}{z}
$$

where we put

$$
\mathcal{R}=z^{2-2 / \nu}-f(z)\left(\dot{v}_{y}\right)^{2}-2 \dot{v}_{y} \dot{z}_{y}
$$

The integrals of motion corresponding to (3.11) read

$$
\begin{aligned}
& \mathcal{J}=-\frac{z^{1-2 / \nu}}{\sqrt{\mathcal{R}}}, \\
& \mathcal{I}=\frac{f(z) \dot{v}_{y}+\dot{z}_{y}}{z \sqrt{\mathcal{R}}} .
\end{aligned}
$$



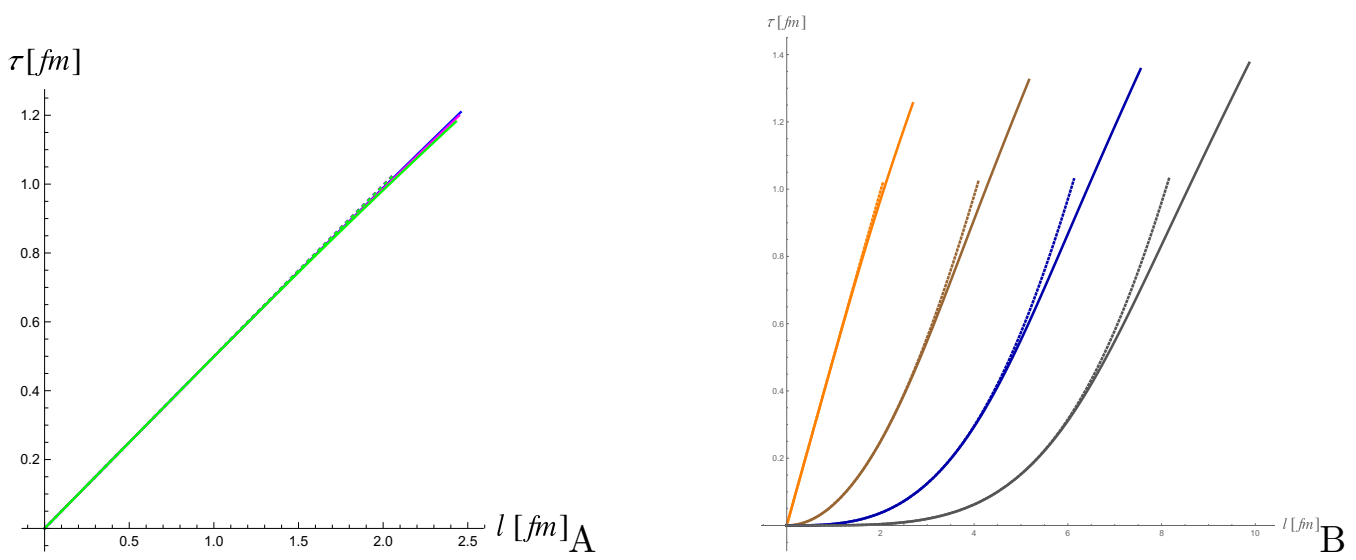

Figure 1. The thermalization time $\tau$ as a function of $\ell$ for the 5-dimensional Lifshitz metric (2.20)-(2.25) for $\nu=2,3,4$. A: thermalization along the longitudinal direction with $m=0.5$ and $m=0.1$. All lines coincide. $B$ : thermalization along the transversal direction, $\nu=1$ (orange), $\nu=2$ (brown), $\nu=3$ (blue) and $\nu=4$ (gray). The solid and dotted curves correspond to $m=0.5$ and $m=0.1$, respectively.

From (3.13) and (3.14) we get that the turning point $z_{*}$ is defined from

$$
-\mathcal{I}^{2} z^{2-\frac{4}{\nu}}\left(f \mathcal{J}^{2} z^{2 / n}-f-\mathcal{I}^{2} z^{2}\right)=0
$$

For $\mathcal{I}=0$ this equation is simplified to give

$$
\mathcal{J}^{2}=\frac{1}{z_{*}^{2 / n}}
$$

and we also get

$$
\dot{z}_{y}= \pm \frac{z^{1-\frac{2}{\nu}} \sqrt{f\left(1-\mathcal{J}^{2} z^{2 / \nu}\right)}}{\mathcal{J}} .
$$

From (3.17) one gets the relation between the ends of geodesic and the thermalization time

$$
\begin{aligned}
\ell & =2 z_{*}^{1 / \nu} \int_{0}^{1} \frac{w^{-1+2 / \nu} d w}{\sqrt{f\left(w z_{*}\right)\left(1-w^{2 / \nu}\right)}}, \\
t_{\text {therm }} & =z_{*} \int_{0}^{1} \frac{d w}{f\left(z_{*} w\right)} .
\end{aligned}
$$

Here we remove the regularization since $\nu>0$. The dependence (3.19) on (3.18) is given in figure 1.B. We see that the thermalization time in the transversal direction depends on the anizotropic parameter $\nu$. In particular, for $\nu=2$ the thermalization process is more then twice faster as compared to the longitudinal direction. By increasing $\nu$ we make the thermalization in the transversal direction faster. We also see that for larger values of $\nu$ the dependence on the mass $m$ becomes more essential. 


\section{Entanglement entropy}

In this section we explore the evolution of entanglement entropy in the context of the holographic prescription. We perform calculations using both black brane (2.13)-(2.14) and Vaidya-Lifshitz time dependent backgrounds (2.24)-(2.25).

The entanglement entropy can be useful to probe correlations in the background measuring an entanglement of a quantum system. If the system is divided into two spatially disjoint parts $A$ and $B$, the entanglement entropy $S(A)$ gives an estimation of the amount of information loss corresponding to the restriction of an $A$. It seems not to be simple to calculate the entanglement entropy from the strongly coupled system side. However, one can compute its holographic dual using the suggestion from works [48]-[50]. The holographic formula for the entanglement entropy of a subsystem $A$

$$
S=\frac{\mathcal{A}}{4 G_{5}},
$$

where $\mathcal{A}$ is the area of the minimal three-dimensional surface whose boundary coincides with the boundary of the region $A$. The area of the surface is defined by the relation

$$
\mathcal{A}=\int d^{3} \sigma \sqrt{\left|\operatorname{det} g_{M N} \partial_{\alpha} X^{M} \partial_{\beta} X^{N}\right|}
$$

where

$$
\sigma^{1}=x, \quad \sigma^{2}=y_{1}, \quad \sigma^{3}=y_{2} .
$$

(4.1) is known as the holographic entanglement entropy. It is useful to represent (2.20)(2.21) and (2.24)-(2.25) in the generic form

$$
g_{M N}=g_{00} d t^{2}+g_{11} d x^{2}+g_{22} d y_{1}^{2}+g_{33} d y_{2}^{2}+g_{44} d z^{2} .
$$

From (4.4) we see that the entanglement entropy depends on the direction along which the subsystem is delineated. There are two possible cases for the subsystems both for the black brane and the thin shell we have we study and compare each other.

\subsection{Entanglement entropy in a time-independent background}

To begin with, we compute the entanglement entropy for the black hole (2.20)-(2.21). Here we present the results for the two subsystems cut out both along longitudinal and transversal directions.

\subsubsection{Subsystem delineated along the longitudinal direction}

First, consider the subsystem $A$ cut out along $x$-direction, say, the belt is located as

$$
x \in\left[0, l_{x}<L_{x}\right], \quad y_{1} \in\left[0, L_{y_{1}}\right], \quad y_{2} \in\left[0, L_{y_{2}}\right] .
$$

We assume that the minimal area surface is invariant under the $y_{1}$ and $y_{2}$ planar directions and the embedding function is the function of only one coordinate, $z=z(x)$. Thus, the three-dimensional minimal surface is defined by

$$
\mathcal{A}=2 \int_{0}^{l_{x}} d x \int_{0}^{L_{y_{1}}} d y_{1} \int_{0}^{L_{y_{2}}} d y_{2} \sqrt{g_{22} g_{33}\left(g_{11}+g_{44}\left(z^{\prime}\right)^{2}\right)} .
$$


Taking into account (2.20), one has

$$
\mathcal{A}=2 L_{y_{1}} L_{y_{2}} \int_{0}^{l_{x}} d x \mathcal{L}, \text { where } \mathcal{L}=\frac{1}{z^{1+2 / \nu}} \sqrt{1+\frac{z^{\prime 2}}{f(z)}}
$$

where it is supposed that $\prime=\frac{d}{d x}$.

The integral of motion corresponding to the system with the Lagrangian $\mathcal{L}$ reads

$$
-\frac{z^{-1-2 / \nu}}{\sqrt{1+\frac{z^{\prime 2}}{f(z)}}}=\mathcal{C} \text {. }
$$

The function $z=z(x)$ that minimizes the surface area is then given by the equation of motion

$$
z^{\prime}= \pm \sqrt{f(z)\left(\left(\frac{z_{*}}{z}\right)^{2(1+2 / \nu)}-1\right)}
$$

where the turning point $z_{*}$ is related with $\mathcal{C}$ as $z_{*}^{1+2 / \nu}=\mathcal{C}^{-1}$. The length scale $l_{x}$ can be found from

$$
\frac{l_{x}}{2}=\int_{z_{0}}^{z_{*}-\epsilon} \frac{d z}{z^{\prime}}=\int_{z_{0}}^{z_{*}-\epsilon}\left(\frac{z}{z_{*}}\right)^{1+\frac{2}{\nu}} \frac{d z}{\sqrt{f(z)\left[1-\left(\frac{z}{z_{*}}\right)^{2(1+2 / \nu)}\right]}}
$$

We can remove $\epsilon$ from the upper limit in (4.10) under the assumption that the turning point $z_{*}$ is above the horizon. Indeed, if the function $f$ is given by (2.21) with the horizon defined by

$$
z_{h}=\frac{1}{m^{\nu /(2+2 \nu)}},
$$

the integrand in (4.10) near $z=z_{*}$ can be represented as

$$
\frac{1}{\sqrt{f\left(1-\frac{z^{2+4 / \nu}}{z_{*}^{2+4 / \nu}}\right)}}=\sqrt{\frac{\nu z_{*}}{2(\nu+2)}} \frac{1}{\sqrt{\left(z_{*}-z\right)\left(1-m z_{*}^{2+\frac{2}{\nu}}\right)}}+\mathcal{O}\left(\sqrt{z-z_{*}}\right)
$$

thus, we have the integrable singularity for $z_{*}<z_{h}$. However, for $z_{*}=z_{h}$ one obtains

$$
\frac{1}{\sqrt{\left(1-\left(\frac{z}{z_{*}}\right)^{2+\frac{2}{\nu}}\right)\left(1-\left(\frac{z}{z_{*}}\right)^{2+\frac{4}{\nu}}\right)}}=\frac{1}{2 \sqrt{\frac{\nu^{2}+3 \nu+2}{\nu^{2} z_{*}^{2}}}\left(z-z_{*}\right)}+\frac{-\nu-3}{4 \nu z_{*} \sqrt{\frac{\nu^{2}+3 \nu+2}{\nu^{2} z_{*}^{2}}}}+O\left(\left(z-z_{*}\right)^{1}\right),
$$

which leads to the logarithmic singularity. For calculations of the entropy in the black hole background we assume that the turning point is below the horizon, while for the case of the shell we present the results for the case when the horizon is crossed.

Substituting (4.9) into (4.7) and coming to the integration with respect to $z$-variable, one has

$$
\mathcal{A}=2 L_{y_{1}} L_{y_{2}} \int_{z_{0}}^{z_{*}} d z \mathfrak{a}(z), \quad \mathfrak{a}(z)=\frac{1}{z^{1+2 / \nu} \sqrt{f(z)\left(1-\left(z / z_{*}\right)^{2(1+2 / \nu)}\right)}} .
$$


In (4.14) we remove $\epsilon$ assuming $m \neq 1$, but keep UV regularization $z_{0}$.

The latter expression can be represented in terms of the dimensionless variable $w=$ $z / z_{*}$ as

$$
\frac{\mathcal{A}}{2 L_{y_{1}} L_{y_{2}}}=\frac{1}{z_{*}^{2 / \nu}} \int_{z_{0}}^{1} \frac{d w}{w^{1+2 / \nu} \sqrt{f\left(z_{*} w\right)\left(1-w^{2(1+2 / \nu)}\right)}} .
$$

The renormalized functional for the minimal surface reads

$$
\frac{\mathcal{A}_{\mathrm{ren}}}{2 L_{y_{1}} L_{y_{2}}}=\frac{1}{z_{*}^{2 / \nu}} \int_{0}^{1} \frac{d w}{w^{1+2 / \nu}}\left[\frac{1}{\sqrt{f\left(1-w^{2+4 / \nu}\right)}}-1\right]-\frac{\nu}{2 z_{*}^{2 / \nu}} .
$$

Taking into account (4.12) one can also rewrite (4.10) in the $w$-variable

$$
l_{x}=2 \int_{0}^{1} z_{*} w^{1+2 / \nu} \frac{d w}{\sqrt{f\left(z_{*} w\right)\left(1-w^{2(1+2 / \nu)}\right)}}
$$

for $z_{*} \neq z_{h}$.

One can see that the relation for the entanglement entropy is proportional to the area of the boundary $\partial A=L_{y_{1}} L_{y_{2}}$ which is in agreement to the area law.

The behavior of the area (4.16) is presented in figure $2 \mathrm{~A}$. To get the dependence of the entanglement entropy of the length for small values of $\ell$ one can consider the massless case. We see that for $m=0$ the integrals (4.16) and (4.17) can be calculated explicitly. By analogy with [25] one gets

$$
\mathcal{A}_{\text {ren }} \propto-\frac{1}{\ell^{2 / \nu}} .
$$

From numerical calculations we see that for large $\ell$

$$
\frac{\mathcal{A}_{\text {ren }}}{L_{y_{1}} L_{y_{2}}} \approx \gamma_{L}(m) \ell+\ldots
$$

To keep the correct dimension we have to assume

$$
\gamma_{L}(m) \propto m^{\frac{2+\nu}{2(1+\nu)}}
$$

It should also be noted, that from figure 2.A the dependence on the mass of the black brane for the intermediate $\ell$ is rather small. The physical meaning of estimation (4.19) is that our surface for large $\ell$ becomes like a smothered parallelepiped almost touching to the horizon.

\subsubsection{Subsystem delineated along the transversal direction}

Another possible subsystem $A$ can be divided along the $y_{1}$-direction (which is equivalent to dividing it along $\left.y_{2}\right)$. It is also assumed that $z=z\left(y_{1}\right)$ and

$$
x \in\left[0, L_{x}\right], \quad y_{1} \in\left[0, l_{y_{1}}<L_{y_{1}}\right], \quad y_{2} \in\left[0, L_{y_{2}}\right] .
$$

The three-dimensional minimal surface bordering on $\partial A$ has the form

$$
\mathcal{A}=2 L_{x} L_{y_{2}} \int_{0}^{l_{y_{1}}} d y_{1} \mathcal{L}, \text { where } \quad \mathcal{L}=\frac{1}{z^{1+1 / \nu}} \sqrt{\frac{1}{z^{2 / \nu}}+\frac{\left(z^{\prime}\right)^{2}}{f(z) z^{2}}} .
$$



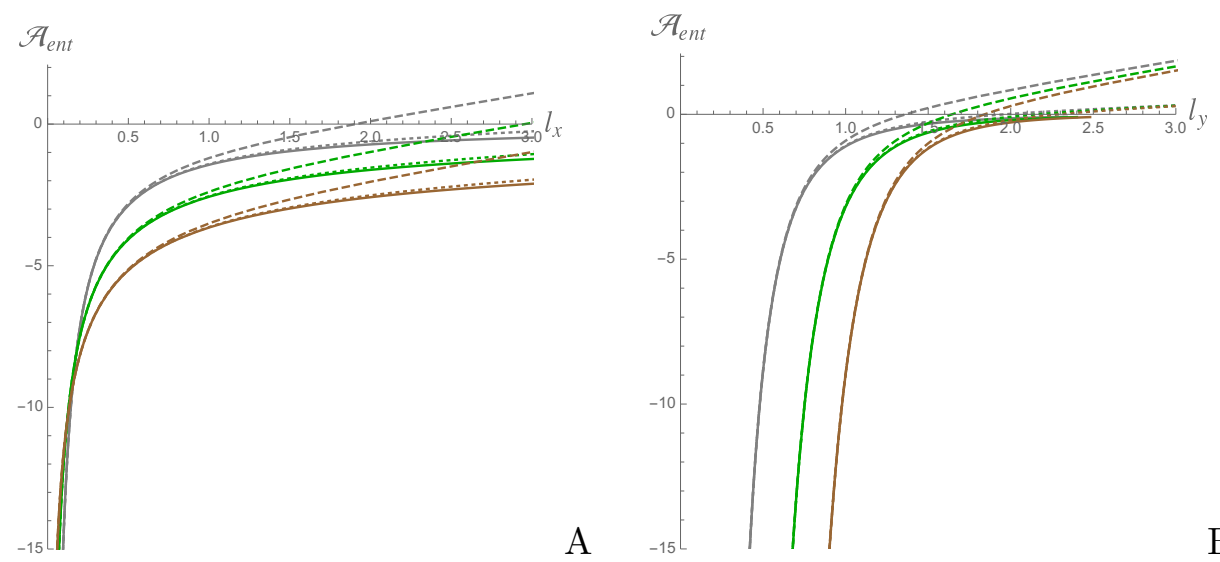

$\mathrm{B}$

Figure 2. $A$ : the $\frac{\mathcal{A}_{\text {ren }}}{2 L_{y_{1}} L_{y_{2}}}$ as a function of $\ell$ (4.17) in the 5 d Lifshitz black brane background (2.20)(2.21) for $\nu=2,3,4$ (the upper gray, middle green and lower brown curves, respectively). $B$ : dependencies of $\frac{\mathcal{A}_{\text {ren }}}{2 L_{x} L_{y_{2}}}$ (4.27) on $\ell$ (4.25) in the black brane (2.20)-(2.21) for $\nu=2,3,4$ (from left to right, respectively). For both cases we plot for $m=0$ (solid lines), $m=0.1$ (dotted lines) and $m=0.9$ (dashed lines).

This dynamical system has the following integral of motion

$$
-\frac{\left.z^{-(1+3 / \nu}\right)}{\sqrt{\frac{1}{z^{2 / \nu}}+\frac{1}{z^{2} f(z)}\left(z^{\prime}\right)^{2}}}=\mathcal{C}
$$

where $\iota=\frac{d}{d y_{1}}$.

The corresponding equation of motion reads

$$
z^{\prime}= \pm z^{1-1 / \nu} \sqrt{f(z)\left(\left(\frac{z}{z_{*}}\right)^{-2(1+2 / \nu)}-1\right)}
$$

where $z_{*}$ is related with $\mathcal{C}$ as $z_{*}^{1+2 / \nu}=\mathcal{C}^{-1}$. The length scale $l_{y_{1}}$ can be defined in the following way

$$
l_{y_{1}}=2 z_{*}^{1 / \nu} \int_{0}^{1} \frac{w^{3 / \nu} d w}{\sqrt{f\left(w, z_{*}\right)\left(1-w^{2(1+2 / \nu)}\right)}} .
$$

We note that for the lower limit in (4.25) one can take $z_{0}=0$. At the same time, we can remove $\epsilon$ for the upper limit of (4.25) for $z_{*}<z_{h}$, by the same reason as above in (4.10).

Owing to (4.24) the relation (4.22) in terms of the dimensionless $w$-variable takes the form

$$
\frac{\mathcal{A}}{2 L_{x} L_{y_{2}}}=\frac{1}{z_{*}^{(1+1 / \nu)}} \int_{z_{0} / z_{*}}^{1} \frac{1}{w^{(2+1 / \nu)}} \frac{1}{\sqrt{f\left(w z_{*}\right)\left(1-w^{2(1+2 / \nu)}\right)}} d w
$$

The renormalized functional for the minimal surface (4.26) reads

$$
\frac{\mathcal{A}_{\mathrm{ren}}}{2 L_{x} L_{y_{2}}}=\frac{1}{z_{*}^{1+1 / \nu}}\left[\int_{0}^{1} \frac{d w}{w^{2+1 / \nu}}\left(\frac{1}{\sqrt{f\left(1-w^{2+4 / \nu}\right)}}-1\right)-\frac{\nu}{1+\nu}\right] .
$$


Numerical results for the entanglement entropy density (4.27) for different values of $\nu$ are shown in figure 2. In a similar way with [25] one can estimate for small $\ell$

$$
\mathcal{A}_{\text {ren }} \propto-\frac{1}{\ell_{y}^{1+\nu}}
$$

and numerical calculations approximately give

$$
\mathcal{A}_{\text {ren }} \approx \gamma_{T}(m) \ell+\ldots
$$

when $\ell$ is large. To keep the correct dimension we have to write

$$
\gamma_{T}(m) \propto m^{\frac{2 \nu+1}{2(1+\nu)}}
$$

for large $\ell$. We see the dependence on the mass in figure 2.B for large $\ell$. Note that the functions $\gamma_{L}(m)(4.19)$ and $\gamma_{T}(m)$ (4.29) are different.

\subsection{Entanglement entropy in a time-dependent background}

Now we come to studies of the evolution of entanglement entropy in the Lifshitz-Vaidya background (2.24)-(2.25), describing the infalling shell. As before we will consider subsystems delineated along both transversal and longitudinal directions.

\subsubsection{Subsystem delineated along the longitudinal direction}

We once again start from the consideration of a subsystem $A$ extending along $x$-direction, assuming that the minimal surface area is parameterized by

$$
v=v(x), \quad z=z(x) .
$$

Taking into account (4.5), the volume functional corresponding to the minimal threedimensional surface is given by

$$
\begin{aligned}
& \mathcal{A}=2 L_{y_{1}} L_{y_{2}} \int_{0}^{l_{x}} d x \mathcal{L}, \\
& \mathcal{L}=\frac{1}{z^{2 / \nu+1}} \sqrt{1-f(z, v)\left(v^{\prime}\right)^{2}-2 v^{\prime} z^{\prime}} .
\end{aligned}
$$

Here we suppose that $I \equiv \frac{d}{d x}$. Substituting (4.32) in (4.1) we get the expression for the holographic entanglement entropy.

The Lagrangian $\mathcal{L}$ in (4.32) has the integral of motion given by

$$
\mathcal{J}=-\frac{1}{z^{1+\frac{2}{\nu}} \sqrt{\mathcal{R}}}
$$

where we denote

$$
\mathcal{R}=1-f(z, v)\left(v^{\prime}\right)^{2}-2 v^{\prime} z^{\prime} .
$$

From (4.34) we immediately obtain

$$
z_{*}^{2+4 / \nu}=z^{2+4 / \nu} \mathcal{R},
$$



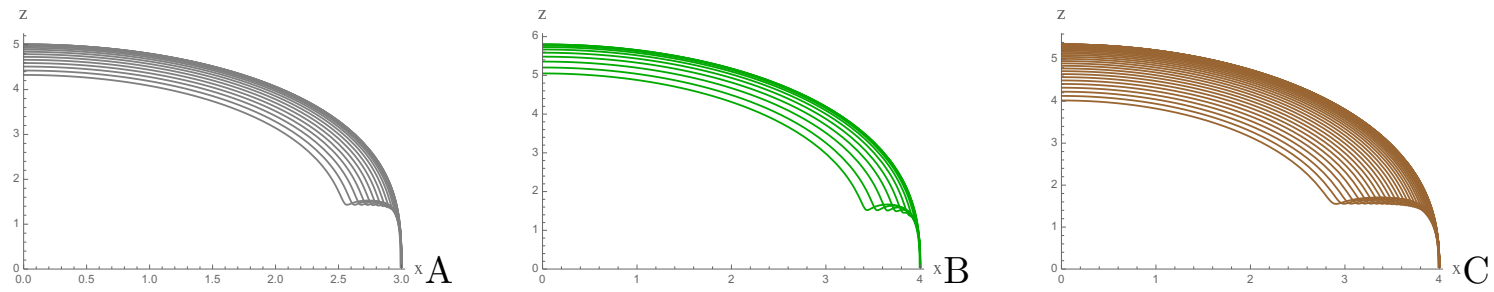

Figure 3. The behavior of the solution for $z(x)$ to eqs. (4.37)-(4.38). A: $\nu=2, z(3)=0$. B: $\nu=3, z(4)=0 . C: \nu=4, z(4)=0$.

where $z_{*}$ is the turning point defined from the requirements $z^{\prime}=v^{\prime}=0$ and related with $\mathcal{J}$ as $z_{*}^{1+2 / \nu}=\mathcal{J}^{-1}$.

The equations of motion following from the Lagrangian $\mathcal{L}(4.33)$ are

$$
\begin{aligned}
2 \nu z v^{\prime \prime}= & \nu z \frac{\partial f}{\partial z} v^{\prime 2}+2(2+\nu)\left(1-f\left(v^{\prime}\right)^{2}-2 v^{\prime} z^{\prime}\right) \\
2 \nu z z^{\prime \prime}= & -2(2+\nu) f+4 f^{2} v^{\prime 2}+2 \nu f^{2} v^{\prime 2}+4(2+\nu) f v^{\prime} z^{\prime}-\nu z v^{\prime 2} \frac{\partial f}{\partial v} \\
& -\nu z f v^{\prime 2} \frac{\partial f}{\partial z}-2 \nu z v^{\prime} z^{\prime} \frac{\partial f}{\partial z}
\end{aligned}
$$

which coincide with those from [51] for $\nu=1$.

Taking into account (4.36) the equations of motion (4.37)-(4.38) can be rewritten as

$$
\begin{aligned}
& 2 \nu z v^{\prime \prime}=\nu z \frac{\partial f}{\partial z} v^{\prime 2}+2(2+\nu) \frac{z_{*}^{2(1+2 / \nu)}}{z^{2(1+2 / \nu)}} \\
& 2 \nu z z^{\prime \prime}=-\left[2(2+\nu) f \frac{z_{*}^{2(1+2 / \nu)}}{z^{2(1+2 / \nu)}}+\nu z v^{\prime 2} \frac{\partial f}{\partial z}+\nu z f v^{\prime 2} \frac{\partial f}{\partial v}+2 \nu z v^{\prime} z^{\prime} \frac{\partial f}{\partial z}\right] .
\end{aligned}
$$

Here we assume that the function $f$ has the form (2.27). We can solve these equation numerically using the following initial conditions

$$
\begin{array}{ll}
z(0)=z_{*}, & z^{\prime}(0)=0 \\
v(0)=v_{*}, & v^{\prime}(0)=0
\end{array}
$$

We are interested in solutions which reach $z=0$ at some point $x_{s}$, similar boundary conditions have been proposed for example in $[52,53]$. The point $x_{s}$ is, in fact, a singular point of the solutions. Solutions to eqs. (4.37)-(4.38) obeying (4.40)-(4.41) are presented in figure 3. We can observe that there are two types of such solutions that have a $z_{*}$ below and above the horizon. It is useful to study a domain of the initial data, where these solutions can exist, see appendix B.

It is also instructive to see the behavior of the quantity $f v^{\prime}+z^{\prime}$. An assumption, that the function $f$ does not depend on $v$, yields to the fact that $f v^{\prime}+z^{\prime}$ is some conserved quantity. At the same time for $f$ defined by (2.27) it changes that we observe on figure 15 . 
However, it can also be seen that at the end of the curve $z=z(x)$ at $x=x_{U V}$, this quantity does not vary significantly and admits the approximation $\partial_{v} f=0$.

Owing to (4.36) the minimal three-dimensional surface (4.32) can be represented as

$$
\mathcal{A}=2 L_{y_{1}} L_{y_{2}} \int_{0}^{\ell_{x}} \frac{d x}{z^{1+2 / \nu}}\left(\frac{z_{*}}{z}\right)^{1+2 / \nu} .
$$

Coming to the $z$-variable one can rewrite (4.42) in the following form

$$
\frac{\mathcal{A}}{2 L_{y_{1}} L_{y_{2}}}=-\int_{z_{0}}^{z_{*}} d z \mathfrak{a}(z)
$$

with $\mathfrak{a}(z)$ defined by

$$
\mathfrak{a}(z)=\frac{1}{z^{\prime} z^{1+2 / \nu}}\left(\frac{z_{*}}{z}\right)^{1+2 / \nu} .
$$

In (4.44) the r.h.s. is taken with the negative sign since $z^{\prime}<0$ for the solutions of our interest.

To calculate the entanglement entropy we have to study the behavior of the integrand $\mathfrak{a}$ in (4.42). For $z \sim 0$ we expect the following behaviour

$$
\mathfrak{a}(z) \sim \frac{1}{z^{1+2 / \nu}}
$$

It is also convenient to introduce the quantity $\mathfrak{b}(z)$, defined by

$$
\mathfrak{b}(z)=\frac{1}{z^{\prime}}\left(\frac{z_{*}}{z}\right)^{1+2 / \nu}
$$

We study the behaviour of function $\mathfrak{b}(z)$ on the solution to eqs. (4.37)-(4.38) for $\nu=2$ and different masses is shown in figure 16 (appendix B). We see that $\mathfrak{b}(z) \rightarrow C \neq 0$ for any value of mass, and therefore, we have $\mathfrak{a}(z) \sim C / z^{1+2 / \nu}$. From figure 16 one can see that for $z_{*}=1$ we have $C=1$. Hence, the UV divergence is similar to the shell free case and one can perform the similar renormalization

$$
\frac{\mathcal{A}_{\mathrm{ren}}^{\text {Shell }}}{2 L_{y_{1}} L_{y_{2}}}=-\left(\int_{z_{0}}^{z_{*}} \frac{\left[\mathfrak{b}(z)-\mathfrak{b}\left(z_{0}\right)\right]}{z^{1+2 / \nu}} d z-\frac{\nu}{2} \frac{\mathfrak{b}\left(z_{0}\right)}{z_{*}^{2 / \nu}}\right) .
$$

Returning to the variable $x$ we obtain the finite contribution to the entanglement entropy of the shell

$$
\frac{\mathcal{A}_{\mathrm{ren}}^{\text {Shell }}}{2 L_{y_{1}} L_{y_{2}}}=\int_{\varepsilon}^{\ell_{x}} \frac{d x}{z^{1+2 / \nu}}\left(\frac{z_{*}}{z}\right)^{1+2 / \nu}-\frac{\nu}{2} \frac{\mathfrak{b}\left(z_{0}\right)}{z_{0}^{2 / \nu}} .
$$

Now we can define the quantity $\Delta \mathcal{A}^{\text {Shell-LV }}$

$$
\frac{\Delta \mathcal{A}^{\text {Shell-LV }}}{2 L_{y_{1}} L_{y_{2}}}=\frac{\mathcal{A}^{\text {Shell }}-\mathcal{A}^{L V}}{2 L_{y_{1}} L_{y_{2}}} .
$$

It should be noted that the holographic entanglement entropy for the Lifshitz-Vaidya background depends on two parameters, $z_{*}$ and $v_{*}$, whereas for the pure Lifshitz case it depends 
only on $z_{*}$. One takes $z_{*}$ in the second term in such a way that it gives the same distance $\ell$ as in the first term. From (4.17) and for $f=1$ one gets

$$
\begin{aligned}
& \nu=2: l_{\text {sing }}=l_{x} / 2 \approx 0.59907 z_{*}, \\
& \nu=3: l_{\text {sing }}=l_{x} / 2 \approx 0.68978 z_{*}, \\
& \nu=4: l_{\text {sing }}=l_{x} / 2 \approx 0.74687 z_{*} .
\end{aligned}
$$

Taking into account that

$$
\frac{\mathcal{A}^{L V}}{2 L_{y_{1}} L_{y_{2}}}=\int_{z_{0}}^{z_{*}} \frac{d z}{z^{1+2 / \nu}} \frac{1}{\sqrt{1-\left(\frac{z}{z_{*}}\right)^{2+4 / \nu}}}=\frac{a_{\nu, \mathrm{ren}}}{z_{*}^{2 / \nu}}+\frac{\nu}{2} \frac{1}{z_{0}^{2 / \nu}},
$$

where $a_{2, \text { ren }}=-0.5991, a_{3, \text { ren }}=-1.03468$, and $a_{4, \text { ren }}=-1.49367$, we can explicitly write down $\Delta \mathcal{A}^{\text {Shell-LV }}$. Thus, we have

$$
\begin{gathered}
\nu=2: \quad \frac{\Delta \mathcal{A}^{\text {Shell-LV }}}{2 L_{y_{1}} L_{y_{2}}}=\int_{\varepsilon}^{\ell_{x}-\epsilon} \frac{z_{*}^{2} d x}{\left(z_{f=f(z, v)}(x)\right)^{4}}-\frac{1}{z_{0}}+\frac{0.5991 \cdot 0.59907}{\ell_{\text {sing }}} \\
\nu=3: \quad \frac{\Delta \mathcal{A}^{\text {Shell-LV }}}{2 L_{y_{1}} L_{y_{2}}}=\int_{\varepsilon}^{\ell_{x}-\epsilon} \frac{z_{*}^{5 / 3} d x}{\left(z_{f=f(z, v)}(x)\right)^{10 / 3}}-\frac{3}{2 z_{0}^{2 / 3}}+\frac{1.03468 \cdot(0.68977)^{2 / 3}}{\ell_{\text {sing }}^{2 / 3}} \\
\nu=4: \quad \frac{\Delta \mathcal{A}^{\text {Shell-LV }}}{2 L_{y_{1}} L_{y_{2}}}=\int_{\varepsilon}^{\ell_{x}-\epsilon} \frac{z_{*}^{3 / 2} d x}{\left(z_{f=f(z, v)}(x)\right)^{3}}-\frac{2}{z_{0}^{1 / 2}}+\frac{1.49367 \cdot(0.74687)^{1 / 2}}{\ell_{\text {sing }}^{1 / 2}}
\end{gathered}
$$

Figure 4 shows the behavior of the entropy density as a function the length $\ell$ for different values of the anisotropic exponent $\nu$. We see that the entanglement entropy increases in a linear regime at small distances like it was observed for the black brane case. The dependence on the critical exponent grows with the reaching the saturation value of the entropy. In figures 5, 6 we demonstrate the evolution of the entanglement entropy in time. Note that in figures 5 we show the difference between the entropy in the current time and the initial value of the entropy at $t=0$, i.e. the value of the entropy in the Lifshitz vacuum. Figures 6 show the difference between the entropy in the current time and the value of the entropy at very large time (time when the thermalization has already taken place), i.e. the difference between the entropy in the current time and thermal entropy. We observe the kink in the evolution which was considered for Lifshitz $(\nu=2)$ and AdS $(\nu=1)$ backgrounds in [33] and [51], respectively. From figures 5, 6 we see that the entanglement entropy increases almost linearly with time. We note that after the saturation point had been reached the entropy flattens out. It should also be mentioned that the saturation is faster for small values of $\ell$ and is almost independent on the anisotropic parameter $\nu$.

\subsubsection{Subsystem delineated along the transversal direction}

Now we turn to the case when a subsystem $A$ is delineated along $y_{1}\left(y_{2}\right)$-direction. Parameterizing the minimal surface are by $v=v\left(y_{1}\right), z=z\left(y_{1}\right)$ with (4.21), we have

$$
\mathcal{A}=2 L_{x} L_{y_{2}} \int_{0}^{l_{y_{1}}} d y_{1} \mathcal{L}
$$





$\mathrm{D}$

Figure 4. The renormalized entanglement entropy at fixed $t=0,0.6,1,1.4,3$, as a function of $\ell$ for a subsystem delineated along the longitudinal direction, $\nu=2,3,4$ ( $A, B, C$, respectively). In $D$ we plot the renormalized entanglement entropy as a function of $\ell$ at $t=0.9$. The different curves correspond to the values $\nu=2,3,4$ from top to bottom.
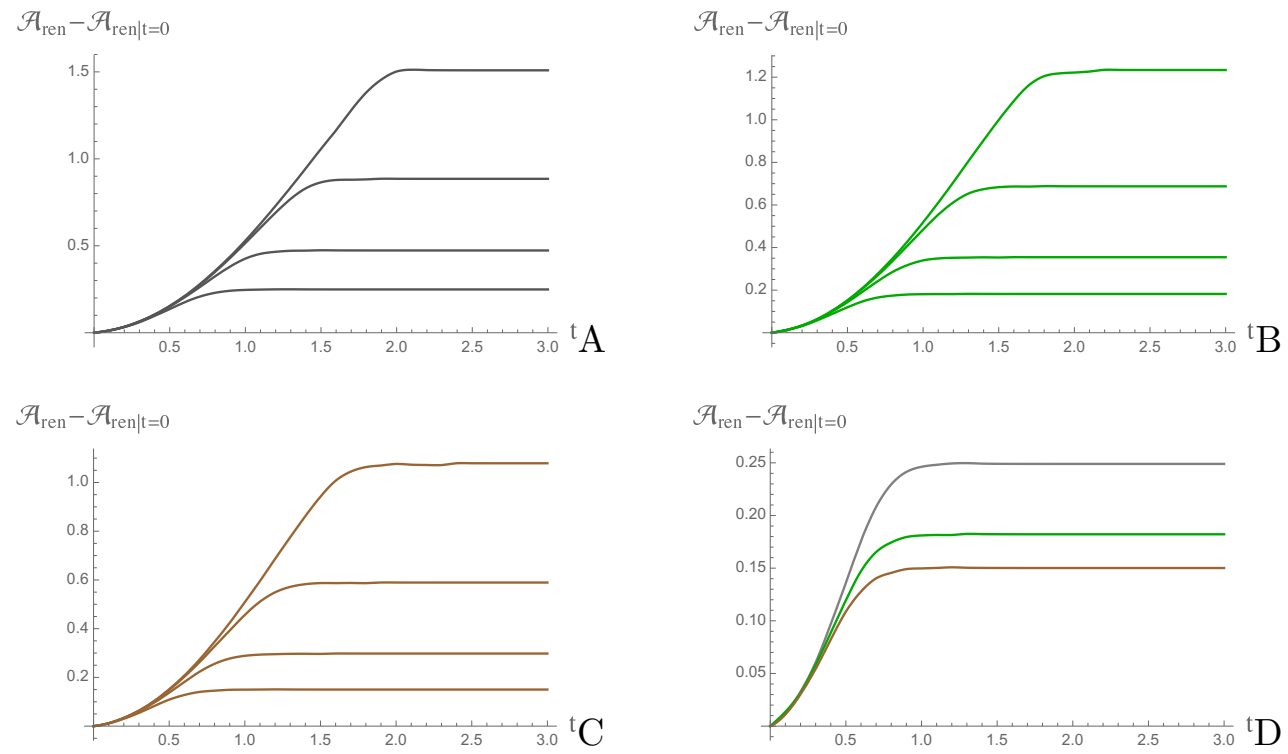

Figure 5. The time dependence of the holographic entanglement entropy $\mathcal{A}_{\text {ren }}$ after the corresponding initial state subtraction $(t=0)$ at fixed $l=1,1.4,2,2.8$ for a subsystem delineated along the longitudinal direction, $\left(A, B, C\right.$, respectively). In $D$ we plot the time dependence of $\mathcal{A}_{\text {ren }}-\left.\mathcal{A}_{\text {ren }}\right|_{t=0}$ at $\ell=1$. The different curves correspond to the values $\nu=2,3,4$ from top to bottom. 


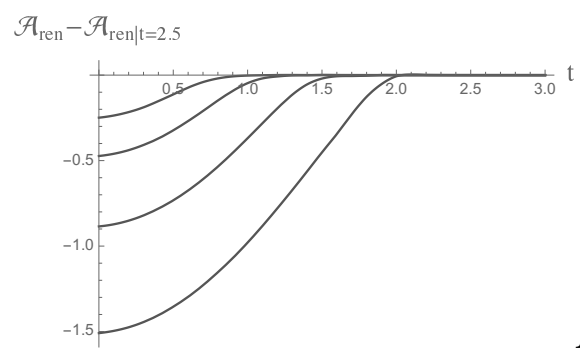

$\mathcal{A}_{\text {ren }}-\mathcal{A}_{\text {ren } \mid \mathrm{t}=2.5}$

A

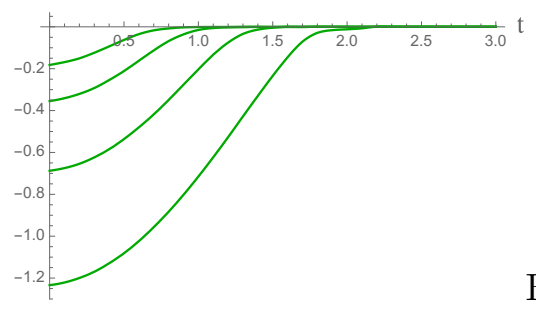

$\mathcal{A}_{\text {ren }}-\mathcal{A}_{\text {ren } \mid \mathrm{t}=2.5}$

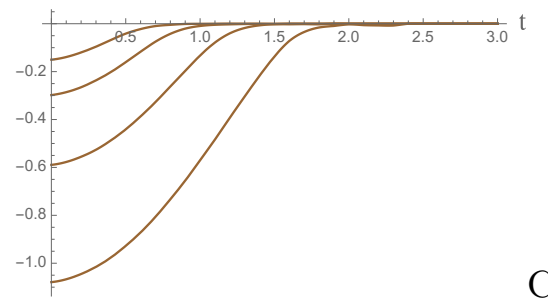

$\mathcal{A}_{\text {ren }}-\mathcal{A}_{\text {ren } \mid \mathrm{t}=2.5}$

$\mathrm{C}$

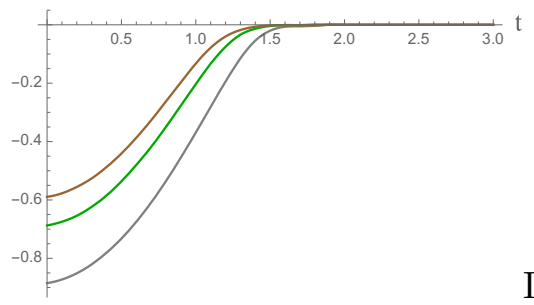

$\mathrm{D}$

Figure 6. The time dependence of the holographic entanglement entropy $\mathcal{A}_{\text {ren }}$ for the LifshitzVaidya metric after the corresponding subtraction of the state when the black brane has already been formed $(t=2.5)$ at fixed $l=1,1.4,2,2.8$ for a subsystem delineated along the longitudinal direction, $\left(A, B, C\right.$, respectively). In $D$ we plot the time dependence of $\mathcal{A}_{\text {ren }}-\left.\mathcal{A}_{\text {ren }}\right|_{t=2.5}$ at $\ell=2$ for the values of $\nu=2,3,4$ from bottom to top.

with

$$
\mathcal{L}=\frac{1}{z^{1+1 / \nu}} \sqrt{\frac{1}{z^{2 / \nu}}-\frac{1}{z^{2}} f(z, v)\left(v^{\prime}\right)^{2}-\frac{2}{z^{2}} v^{\prime} z^{\prime}},
$$

where it is supposed that $I \equiv \frac{d}{d y_{1}}$.

The integral of motion corresponding to the system with Lagrangian $\mathcal{L}(4.57)$ is

$$
\mathcal{J}=-\frac{1}{z^{1+3 / \nu} \sqrt{\mathcal{R}}}
$$

with

Denoting

$$
\mathcal{R}=\frac{1}{z^{2 / \nu}}-\frac{1}{z^{2}} f(z, v)\left(v^{\prime}\right)^{2}-\frac{2}{z^{2}} v^{\prime} z^{\prime} .
$$

we get the conserved quantity

$$
\mathcal{J}=-\frac{1}{z_{*}^{1+2 / \nu}},
$$

$$
z^{6 / \nu}\left(z^{2-2 / \nu}-v^{\prime 2} f-2 z^{\prime} v^{\prime}\right)=z_{*}^{2+4 / \nu} .
$$

The EOM corresponding to (4.57) can be presented in the form

$$
\begin{aligned}
2 \nu z^{1+\frac{2}{\nu}} v^{\prime \prime}= & \nu z^{1+\frac{2}{\nu}} \frac{\partial f}{\partial z} v^{\prime 2}-2 f z^{2 / \nu} v^{\prime 2} \\
& -4 f \nu z^{2 / \nu} v^{\prime 2}-4 \nu z^{2 / \nu} v^{\prime} z^{\prime}-8 z^{2 / \nu} v^{\prime} z^{\prime}+2 \nu z^{2}+4 z^{2} \\
-2 \nu z^{1+\frac{2}{\nu}} z^{\prime \prime}= & -2 f^{2} z^{2 / \nu} v^{\prime 2}-4 \nu f^{2} z^{2 / \nu} v^{\prime 2}+\nu z^{1+2 / \nu} \frac{\partial f}{\partial v} v^{\prime 2}+\nu z^{1+2 / \nu} f \frac{\partial f}{\partial z} v^{\prime 2} \\
- & 4 f z^{2 / \nu} v^{\prime} z^{\prime}-8 \nu f z^{2 / \nu} v^{\prime} z^{\prime}+2 \nu z^{1+2 / \nu} \frac{\partial f}{\partial z} v^{\prime} z^{\prime}+4 z^{2 / \nu} z^{\prime 2}-4 \nu z^{2 / \nu} z^{\prime 2}+4 f z^{2}+2 \nu f z^{2} .
\end{aligned}
$$



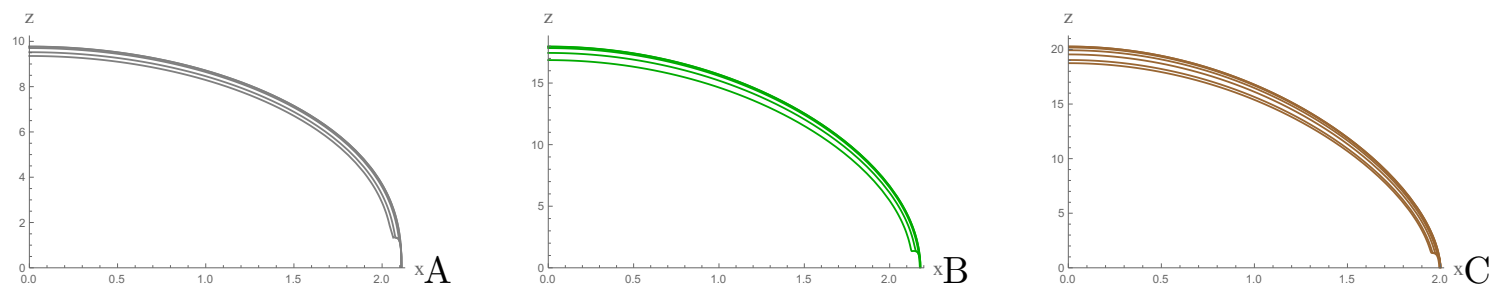

Figure 7. The behavior of the profiles of the solution $z(x)$ to $(4.37)-(4.38)$. A: $\nu=2, z(2.1)=0$. $B: \nu=3, z(2.2)=0 . C: \nu=4, z(2.1)=0$.

Taking into account (4.62) the equations of motion (4.63)-(4.65) can be re written as

$$
\begin{aligned}
2 \nu z^{1+\frac{2}{\nu}} v^{\prime \prime}= & \nu z^{\frac{2}{\nu}+1} \frac{\partial f}{\partial z} v^{\prime 2}+2(2+\nu) z^{2} \frac{z_{*}^{2(1+2 / \nu)}}{z^{2(1+2 / \nu)}}+2(1-\nu) f z^{2 / \nu} v^{\prime 2}, \\
-2 \nu z^{1+\frac{2}{\nu}} z^{\prime \prime}= & 2(2 \nu+1) f z^{2} \frac{z_{*}^{2(1+2 / \nu)}}{z^{2(1+2 / \nu)}}+\nu z^{1+\frac{2}{\nu}} v^{\prime 2} \frac{\partial f}{\partial z}+\nu z^{1+\frac{2}{\nu}} f v^{\prime 2} \frac{\partial f}{\partial v} \\
& +2 \nu z^{1+\frac{2}{\nu}} v^{\prime} z^{\prime} \frac{\partial f}{\partial z}+4(1-\nu) z^{2 / \nu} z^{\prime 2} .
\end{aligned}
$$

Here we assume that the function $f$ is given by (2.27) as in the previous section and we solve (4.63)-(4.65) with the same boundary conditions

$$
\begin{aligned}
& z(0)=z_{*}, \quad z^{\prime}(0)=0, \\
& v(0)=v_{*}, \quad v^{\prime}(0)=0 .
\end{aligned}
$$

We consider once again only solutions that reach $z=0$ at some point $x_{s}$, that is in fact a singular point of the solutions. In figure 7 we plot solutions to (4.63)-(4.65) with (4.67)(4.68). We also present domains of the initial data plane, where these solutions can exist in figure 21-23, see appendix B. We show the position of the singular point corresponding to the solution with given $z_{*}$ and varying $v_{*}$. We present more details about solutions to eqs. (4.37)-(4.38) in appendix B.

One can rewrite (4.57) in the following way

$$
\mathcal{A}=2 L_{x} L_{y_{2}} \int_{0}^{l_{y_{1}}} d y_{1} \frac{z_{*}^{1+2 / \nu}}{z^{2+4 / \nu}}
$$

On the solution $z$ the functional (4.69) can be presented as

$$
\frac{\mathcal{A}}{2 L_{x} L_{y_{2}}}=-\int_{z_{0}}^{z_{*}} d z \mathfrak{a}(z),
$$

where

$$
\mathfrak{a}(z)=\frac{1}{z^{2+1 / \nu}} \mathfrak{b}(z),
$$

and

$$
\mathfrak{b}(z)=\frac{z_{*}^{1+2 / \nu}}{z^{\prime} z^{3 / \nu}}
$$



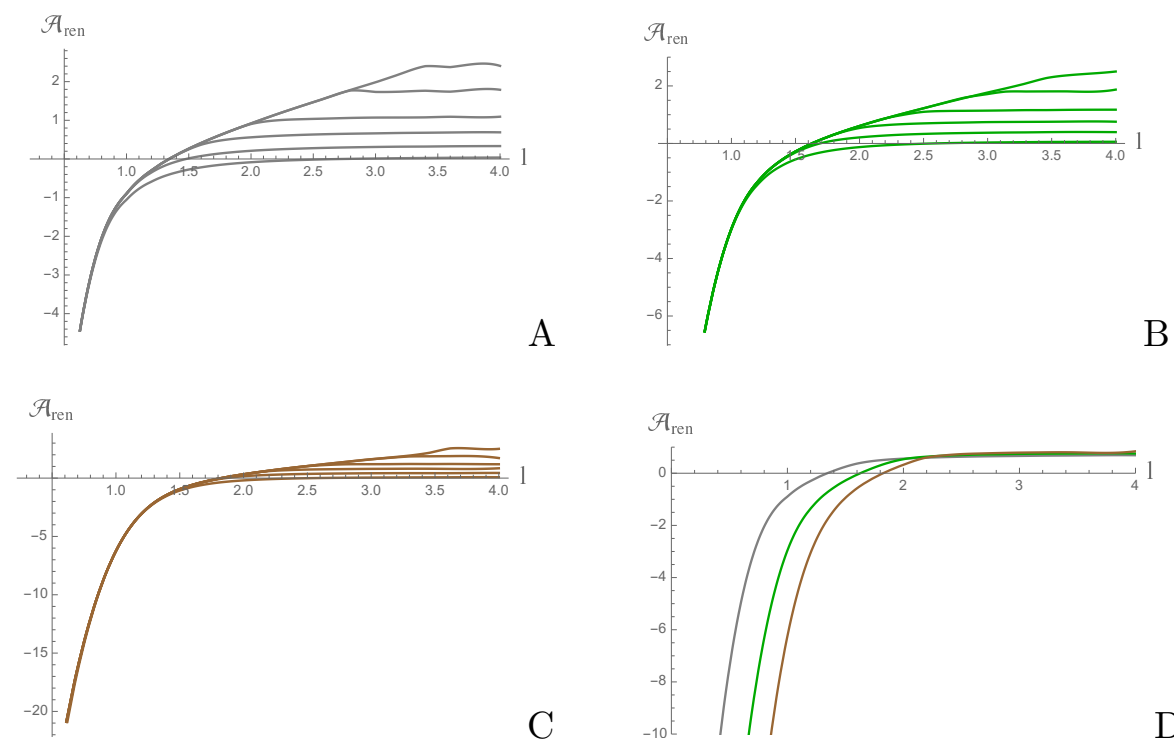

Figure 8. The renormalized entanglement entropy at fixed $t=0.1,0.6,1,1.4,2,2.6$, as a function of $\ell$ for a subsystem delineated along the transversal direction, $\nu=2,3,4(A, B$, $C$, respectively). In $D$ we plot the renormalized entanglement entropy as a function of $l$ at $t=1$ for different values of $\nu$ (from left to right, respectively).

As in the previous section we derive the factor $\mathfrak{b}(z)$ thus $\mathfrak{b}(z) \rightarrow 1$ (see, appendix B). In this case the UV behaviour is the same as in the vacuum case and we can represent the answer to (4.69) in the following form

$$
\frac{\mathcal{A}_{\mathrm{ren}}^{\text {Shell }}}{2 L_{x} L_{y_{2}}}=-\left(\int_{z_{0}}^{z_{*}} \frac{\left[\mathfrak{b}(z)-\mathfrak{b}\left(z_{0}\right)\right]}{z^{2+1 / \nu}} d z-\frac{\nu}{1+\nu} \frac{\mathfrak{b}\left(z_{0}\right)}{z_{*}^{1+1 / \nu}}\right) .
$$

The finite contribution to the holographic entanglement entropy can be represented in the following way

$$
\frac{\mathcal{A}_{\mathrm{ren}}^{\text {Shell }}}{2 L_{x} L_{y_{2}}}=\int_{\epsilon}^{l_{x}} \frac{d x}{z^{2+1 / \nu}} \frac{z_{*}^{1+2 / \nu}}{z^{3 / \nu}}-\frac{\nu}{\nu+1} \frac{\mathfrak{b}\left(z_{0}\right)}{z_{0}^{1+1 / \nu}} .
$$

The renormalized entanglement entropy (4.74) as a funciton of $\ell$ is presented in figure 8 . From figure $2 B$ and figure $8 D$ one can see the entanglement entropy in time-dependent background has the similar behavior as for the static case. For small $\ell$ we observe the dependence of the entropy on $\nu$, which vanishes for large $\ell$, where the entropy has linear behavior.

In three left panels of figure 11 we present the renormalized entanglement entropy (4.74) as a function of $\ell$ and $t$.

Now as above let us define $\Delta \mathcal{A}_{\text {reg }}^{\text {Shell-LV by }}$

$$
\frac{\Delta \mathcal{A}^{\text {Shell-LV }}}{2 L_{x} L_{y_{2}}}=\frac{\mathcal{A}^{\text {Shell }}-\mathcal{A}^{L V}}{2 L_{x} L_{y_{2}}} .
$$

Taking into account that for $\mathcal{A}_{\text {reg }}^{L V}$ we have

$$
\frac{\mathcal{A}^{L V}}{2 L_{x} L_{y_{2}}}=\frac{\nu}{1+\nu} \frac{1}{z_{0}^{1+1 / \nu}}+\left(\frac{L(\nu, 0)}{l_{y_{1}}}\right)^{\nu+1} a_{\nu, \mathrm{ren}},
$$


where $a_{2 \text {,ren }}=-0.225, a_{3 \text {,ren }}=-0.208, a_{4, \text { ren }}=-1.885$ and $L(\nu, 0)$ is read from (4.25) with $m=0$, so

$$
\begin{aligned}
& \nu=2: L(2,0) \approx 0.67497 \\
& \nu=3: L(3,0) \approx 0.8324 \\
& \nu=4: L(4,0) \approx 0.9425
\end{aligned}
$$

Now we get

$$
\frac{\Delta \mathcal{A}^{\text {Shell-LV }}}{2 L_{x} L_{y_{2}}}=\int_{\varepsilon}^{\ell_{y}} \frac{z_{*}^{1+2 / \nu} d x}{\left(z_{f=f(z, v)}(x)\right)^{2+4 / \nu}}-\frac{\nu}{1+\nu} \frac{1}{\left(z\left(\ell_{y}\right)\right)^{\frac{1+\nu}{\nu}}}-\left(\frac{L(\nu, 0)}{l_{y_{1}}}\right)^{\nu+1} a_{\nu, \mathrm{ren}}
$$

For $\nu=2,3,4$ one can write down explicitly

$$
\begin{aligned}
& \nu=2: \frac{\Delta \mathcal{A}^{\text {Shell-LV }}}{2 L_{x} L_{y_{2}}}=\int_{\varepsilon}^{\ell_{y}} \frac{z_{*}^{2} d x}{\left(z_{f=f(z, v)}(x)\right)^{4}}-\frac{2}{3} \frac{1}{z_{0}^{\frac{3}{2}}}+\frac{0.225 \cdot 0.6749^{3}}{l_{y}^{3}}, \\
& \nu=3: \frac{\Delta \mathcal{A}^{\text {Shell-LV }}}{2 L_{x} L_{y_{2}}}=\int_{\varepsilon}^{\ell_{y}} \frac{z_{*}^{5 / 3} d x}{\left(z_{f=f(z, v)}(x)\right)^{10 / 3}}-\frac{3}{4} \frac{1}{z_{0}^{\frac{4}{3}}}+\frac{0.208 \cdot 0.8324^{4}}{l_{y}^{4}} \\
& \nu=4: \quad \frac{\Delta \mathcal{A}^{\text {Shell-LV }}}{2 L_{x} L_{y_{2}}}=\int_{\varepsilon}^{\ell_{y}} \frac{z_{*}^{3 / 2} d x}{\left(z_{f=f(z, v)}(x)\right)^{3}}-\frac{4}{5} \frac{1}{z_{0}^{\frac{5}{4}}}+\frac{0.1885 \cdot 0.9425^{5}}{l_{y}^{5}} .
\end{aligned}
$$

We present the time evolution of the entanglement entropy (4.74) for different values of the critical exponent $\nu$ in figure 9 and figure 10 . In figures 9 we show the difference between the entropy in the shell background and the value of the entropy in the Lifshitz vacuum. The evolution in time of the quantity which represents the difference between the entropy in the current time and thermal entropy is demonstrated in figures 10 . For each value of $\ell$ we observe that the entropy grows linearly at small times. Then it approaches saturation and we see a kink in the dependence on time, which is much sharper for greater values $\ell$. We note that the evolution of the entanglement entropy has more essential dependence on the anisotropic parameter $\nu$ comparing to the case when the subsystem cut out along the longitudinal direction.

Three right panels in figure 11 also demonstrate the behavior of the entanglement entropy with subtracted vacuum values as a function of $\ell$ and $t$ for different $\nu$.

\section{Conclusions}

In this paper, we have investigated the holographic thermalization process of the quarkgluon plasma in anisotropic backgrounds. For this purpose, we have used an analytic black brane solution which asymptotes to the Lifshitz-like spacetime with arbitrary critical exponent. We also have built the corresponding Lifshitz-Vaidya solution, which metric interpolates between the vacuum Lifshitz-like and the black brane geometries. This background has been used to describe the thermalization process as well to model the "quench" 

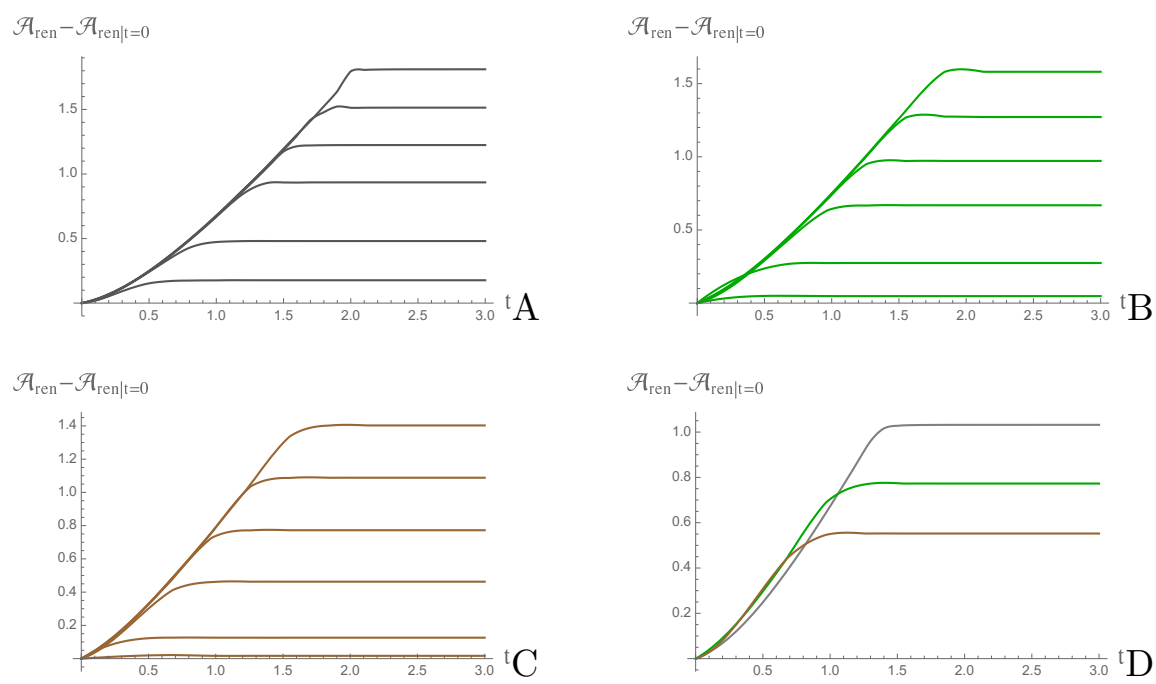

Figure 9. The time dependence of the holographic entanglement entropy $\mathcal{A}_{\text {ren }}$ after the corresponding initial state subtraction for the Lifshitz-Vaidya metric at fixed $l=1,1.4,1.9,2.2,2.5,2.8$ for a subsystem delineated along the transversal direction, $\nu=2,3,4(A, B, C$, respectively). In $D$ we plot the time dependence of $\mathcal{A}_{\text {ren }}-\left.\mathcal{A}_{\text {ren }}\right|_{t=0}$ at $l=2$ for $\nu=2,3,4$ (from top to bottom, respectively)



A
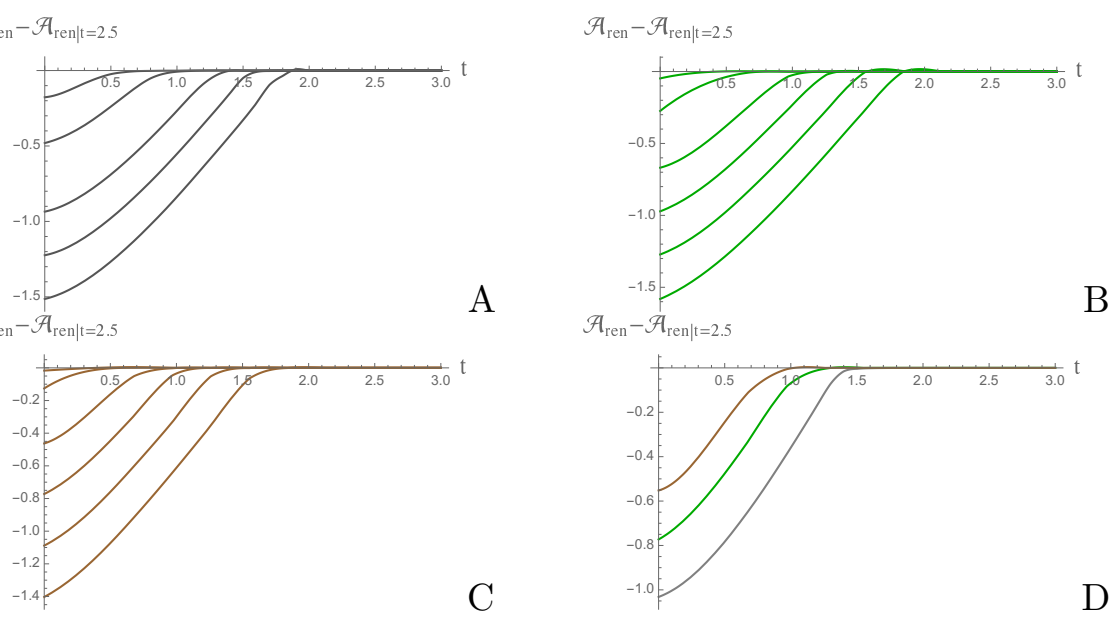

$\mathcal{A}_{\text {ren }}-\mathcal{A}_{\text {ren } \mid \mathrm{t}=2.5}$

B

$\mathrm{C}$

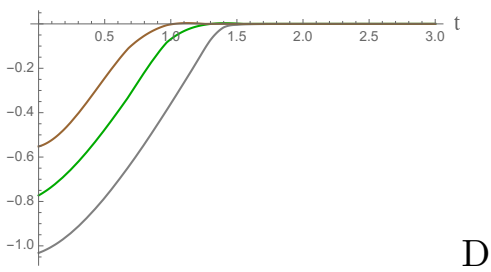

Figure 10. The time dependence of the holographic entanglement entropy $\mathcal{A}_{\text {ren }}$ for the LifshitzVaidya metric after the corresponding subtraction of the state when the black brane has already been formed $(t=2.5)$ at fixed $l=1,1.4,1.9,2.2,2.5,2.8$ for a subsystem delineated along the transversal direction, $\nu=2,3,4$ ( $A, B, C$, respectively). In $D$ we plot the time dependence of $\mathcal{A}_{\text {ren }}-\left.\mathcal{A}_{\text {ren }}\right|_{t=2.5}$ at $l=2$ for $\nu=2,3,4$ (from bottom to top, respectively). 



Figure 11. Left panel: the evolution of $\mathcal{A}_{\text {ren }}^{\text {Shell }}$ on $\ell$ and $t$ for $\nu=2,3,4$ (from top to bottom, respectively); right panel: the evolution of $\Delta \mathcal{A}^{\text {Shell-LV }}$ on $\ell$ and $t$ for $\nu=2,3,4$ (from top to bottom, respectively). 
process. Let us note that 4-dimensional Lifshitz spacetimes with black hole are widely used in AdS/CMT models [54-56].

We have considered thermalization processes both in the transversal and longitudinal directions, which differ by the contribution of the anisotropic exponent. The thermalization along the longitudinal direction turned to have the linear regime similar to that in modified AdS models. At the same time, in the transversal direction the thermalization process is much faster and behaves linearly only for large distances. It should also be noted that the thermalization along the longitudinal direction is independent of the value of the dynamical exponent, while results obtained for the transversal direction strongly depend on the anisotropic parameter and are more sensitive to the value of mass.

Holographic entanglement entropy have also been studied for the subsystems delineated along both transversal and longitudinal directions. For a subsystem cutting out along the longitudinal direction in the black brane background, we have found that the dependence of the entropy on the critical exponent for small distances was absent and appeared for larger values of $\ell$. In the transversal direction we have observed that the entropy depends on $\nu$ at small distances and approaches a linear behavior which is the same for all $\nu$. Thus, for both subsystems at large $\ell$, the entanglement entropy comes to a linear regime, which, depending on the chosen direction, depends or does not on the critical exponent.

The regime is similar to the one found for the Lifshitz metrics in [33], which, however, is independent on $\nu$. This is related to that the anisotropy between the spatial coordinates is absent in the Lifshitz backgrounds considered in [33] unlike the Lifshitz-like metrics suggested in $[22,25]$.

The most interesting results concern the holographic entanglement entropy in the Vaidya-Lifshitz solution that we constructed. Here we again studied subsystems divided along two possible directions. The common feature of the time evolution of the entropy for both subsystems is the kink observed already for small distances. The entropy increases linearly in time until it approaches the saturation point. We found that the form of the kink is sharper for large values of $\ell$. The dependence on the critical exponent looks similar to this one in the black brane background. Since the subsystems differ by the contribution of the critical exponent, the rate of approaching saturation and the saturation value of the entanglement entropy were seen to be different for each case.

It would be interesting to study other non-local operators, like two-point correlation functions and Wilson loops operators, in the backgrounds considered in this paper and compare their velocity bounds as well as estimate with experimental data. We shall address these problems in our future work [27].

\section{Acknowledgments}

We would like to thank Dima Ageev, Misha Khramtsov and Giuseppe Policastro for useful discussions, as well as Blaise Goutreaux and Elias Kiritsis for the correspondence at early stage of the work. We also thank to the JHEP referee for careful reading of our paper and fruitful discussions. This work was supported by the RFBR grant 14-01-00707 and by the ANR grant ANR-12-BS01-012-01. I. A. and A.G. thank the Galileo Galilei Institute 
for Theoretical Physics for the hospitality and the INFN for partial support during the preparation of this work.

\section{A Einstein equations}

Here we provide both sides of the Einstein equations derived from the action (2.4):

$$
R_{m n}=-\frac{\Lambda}{3} g_{m n}+\frac{1}{2} \partial_{m} \phi \partial_{n} \phi+\frac{1}{4} e^{\lambda \phi}\left(2 F_{m p} F_{n}{ }^{p}\right)-\frac{1}{12} e^{\lambda \phi} F^{2} g_{m n} .
$$

The computations have been checked with SageManifolds [28], which is an extension of the free computer algebra system SageMath [29]. The corresponding worksheets are publicly available at the following links:

https://cloud.sagemath.com/3edbca82-97d6-41b3-9b6f-d83ea06fc1e9/raw/Lifshitz_black_brane.html https://cloud.sagemath.com/3edbca82-97d6-41b3-9b6f-d83ea06fc1e9/raw/Vaidya-Lifshitz.html

\section{A.1 The l.h.s. of the Einstein equations}

Without any black brane, the metric reads

$$
d s^{2}=e^{2 \nu r}\left(-d t^{2}+d x^{2}\right)+e^{2 r}\left(d y_{1}^{2}+d y_{2}^{2}\right)+d r^{2} .
$$

The non-zero components of the Ricci tensor are

$$
\begin{aligned}
& R_{00}=2\left(\nu^{2}+\nu\right) e^{2 \nu r}, \quad R_{11}=-2\left(\nu^{2}+\nu\right) e^{2 \nu r} ; \\
& R_{22}=-2(\nu+1) e^{2 r} \quad R_{33}=-2(\nu+1) e^{2 r}, \quad R_{44}=-2\left(\nu^{2}+1\right) \text {. }
\end{aligned}
$$

The scalar curvature is

$$
R=-6 \nu^{2}-8 \nu-6 .
$$

The metric for a black brane solution that asymptotes to the Lifshitz background (A.2) is given by

$$
d s^{2}=e^{2 \nu r}\left(-f(r) d t^{2}+d x^{2}\right)+e^{2 r}\left(d y_{1}^{2}+d y_{2}^{2}\right)+\frac{d r^{2}}{f(r)}
$$

where

$$
f(r)=1-m e^{-(2 \nu+2) r} .
$$

The geometry (A.6)-(A.7) is supported by

$$
e^{\lambda \phi}=\mu e^{4 r}, \quad F_{(2)}=\frac{1}{2} q d y_{1} \wedge d y_{2} .
$$

The non-zero components of the Ricci tensor of the metric (A.6) are

$$
\begin{aligned}
& R_{00}=e^{2 \nu r} f(r)\left(2\left(\nu^{2}+\nu\right) f(r)+(2 \nu+1) \frac{\partial f(r)}{\partial r}+\frac{1}{2} \frac{\partial^{2} f(r)}{\partial r^{2}}\right) \\
& R_{11}=-2\left(\nu^{2}+\nu\right) e^{2 \nu r} f(r)-\nu e^{2 \nu r} \frac{\partial f(r)}{\partial r} \\
& R_{22}=-2(\nu+1) e^{2 r} f(r)-e^{2 r} \frac{\partial f(r)}{\partial r} \\
& R_{33}=-2(\nu+1) e^{2 r} f(r)-e^{2 r} \frac{\partial f(r)}{\partial r} \\
& R_{44}=-2\left(\nu^{2}+1\right)-\frac{1}{f(r)}(2 \nu+1) \frac{\partial f(r)}{\partial r}-\frac{1}{2 f(r)}\left(\frac{\partial^{2} f(r)}{\partial r^{2}}\right) .
\end{aligned}
$$


The generalization of (A.6)-(A.7) to the Vaidya background reads

$$
d s^{2}=-e^{2 \nu r} f(v, r) d v^{2}+2 e^{\nu r} d v d r+e^{2 \nu r} d x^{2}+e^{2 r}\left(d y_{1}^{2}+d y_{2}^{2}\right),
$$

where

$$
f(v, r)=1-m(v) e^{-(2 \nu+2) r} .
$$

The solution (A.14)-(A.15) is supported by the fields (A.8), plus the infalling shell of null dust, whose energy-momentum tensor is

$$
T^{\mathrm{s}}=T_{00}^{\mathrm{s}} d v \otimes d v .
$$

The non-zero components of the Ricci tensor of the metric (A.14) are

$$
\begin{aligned}
R_{00}= & e^{2 \nu r} f(v, r)\left(2\left(\nu^{2}+\nu\right) f(v, r)+(2 \nu+1) \frac{\partial f(v, r)}{\partial r}+\frac{1}{2} \frac{\partial^{2} f(v, r)}{\partial r^{2}}\right) \\
& -\frac{(\nu+2)}{2} e^{\nu r} \frac{\partial f(v, r)}{\partial v}, \\
R_{04}= & -e^{\nu r}\left(2\left(\nu^{2}+\nu\right) f(v, r)+(2 \nu+1) \frac{\partial f(v, r)}{\partial r}+\frac{1}{2} \frac{\partial^{2} f(v, r)}{\partial r^{2}}\right), \\
R_{11}= & -e^{2 \nu r} \nu\left(2(\nu+1) f(v, r)+\frac{\partial f(v, r)}{\partial r}\right), \\
R_{22}= & R_{33}=-e^{2 r}\left(2(\nu+1) f(v, r)+\frac{\partial f(v, r)}{\partial r}\right), \quad R_{44}=2(\nu-1) .
\end{aligned}
$$

\section{A.2 The r.h.s. of the Einstein equations}

Here we write down the right-hand sides of Einstein equations corresponding to the Vaidya solution (A.14) which asymptotes to the Lifshitz-like spacetime (A.2):

$$
\begin{aligned}
& \mathbf{0 0}:-\frac{\Lambda}{3} g_{00}-\frac{1}{6} e^{\lambda \phi} F_{23} F_{23} g^{22} g^{33} g_{00}+T_{00}^{\mathrm{s}}=\left(\frac{\Lambda}{3}+\frac{1}{24} \mu q^{2}\right) e^{2 \nu r} f(r)+T_{00}^{\mathrm{s}}, \\
& \mathbf{1 1}:-\frac{\Lambda}{3} g_{11}-\frac{1}{6} e^{\lambda \phi} F_{23} F_{23} g^{22} g^{33} g_{11}=-\left(\frac{\Lambda}{3}+\frac{1}{24} \mu q^{2}\right) e^{2 \nu r} \\
& \mathbf{2 2}:-\frac{\Lambda}{3} g_{22}+\frac{1}{2} e^{\lambda \phi} F_{23} F_{23} g^{33}-\frac{1}{6} e^{\lambda \phi} F_{23} F_{23} g^{22} g^{33} g_{22}=-\left(\frac{\Lambda}{3}-\frac{1}{12} \mu q^{2}\right) e^{2 r} \\
& \mathbf{3 3}:-\frac{\Lambda}{3} g_{33}+\frac{1}{2} e^{\lambda \phi} F_{23} F_{23} g^{22}-\frac{1}{6} e^{\lambda \phi} F_{23} F_{23} g^{22} g^{33} g_{33}=-\left(\frac{\Lambda}{3}-\frac{1}{12} \mu q^{2}\right) e^{2 r} \\
& \mathbf{4 4}:-\frac{\Lambda}{3} g_{44}+\frac{1}{2}\left(\partial_{4} \phi\right)^{2}-\frac{1}{6} e^{\lambda \phi} F_{23} F_{23} g^{22} g^{33} g_{44}=\frac{1}{2}\left(\frac{\partial \phi}{\partial r}\right)^{2} \\
& \mathbf{0 4}:-\frac{\Lambda}{3} g_{04}-\frac{1}{6} e^{\lambda \phi} F_{23} F_{23} g^{22} g^{33} g_{04}=-\left(\frac{\Lambda}{3}+\frac{1}{24} \mu q^{2}\right) e^{\nu r} .
\end{aligned}
$$

Substituting (A.15) into (A.17)-(A.26) and representing the non-vanishing term in the component $R_{00}$ (A.17) as

$$
-\frac{(\nu+2)}{2} e^{\nu r} \frac{\partial f(v, r)}{\partial v}=\frac{(\nu+2)}{2} e^{-(\nu+2) r} \frac{d m}{d v},
$$



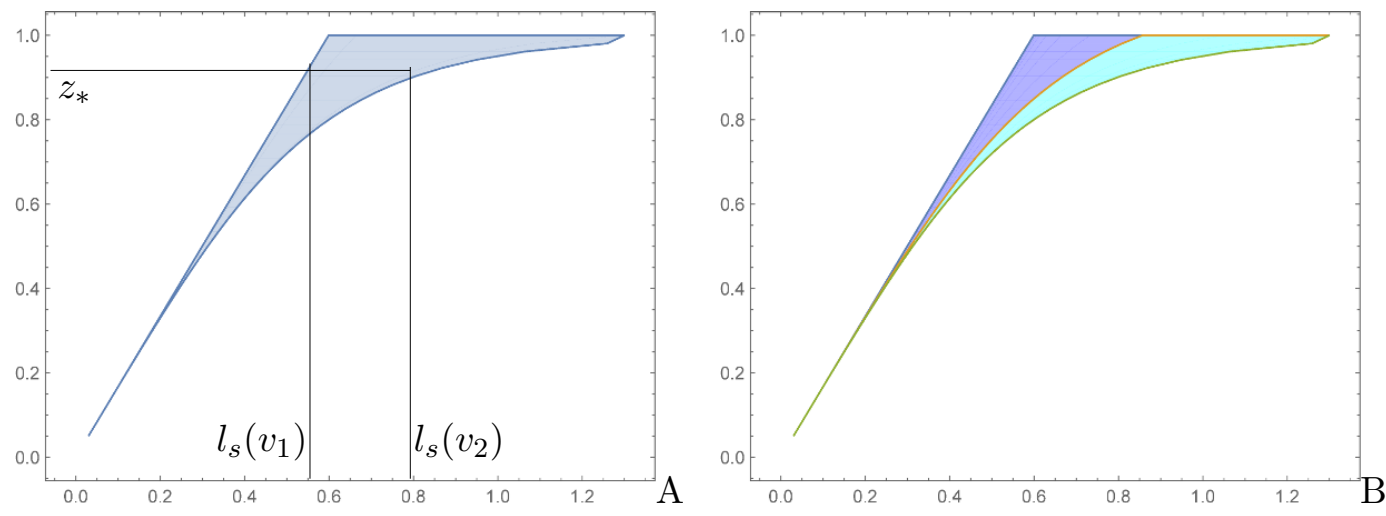

Figure 12. $A$ : positions of the singular point for given $z_{*}$ and given $v_{*}$ belonging to the variety $v_{1} \leq v_{*} \leq v_{2}, l_{s}\left(v_{1}\right) \leq l_{s}\left(v_{1}\right) \leq l_{s}\left(v_{2}\right)$. In this plot $v_{1}=-5, v_{2}=25 . \nu=2$. B: different zones corresponds to different domains for varying $v_{*}$.
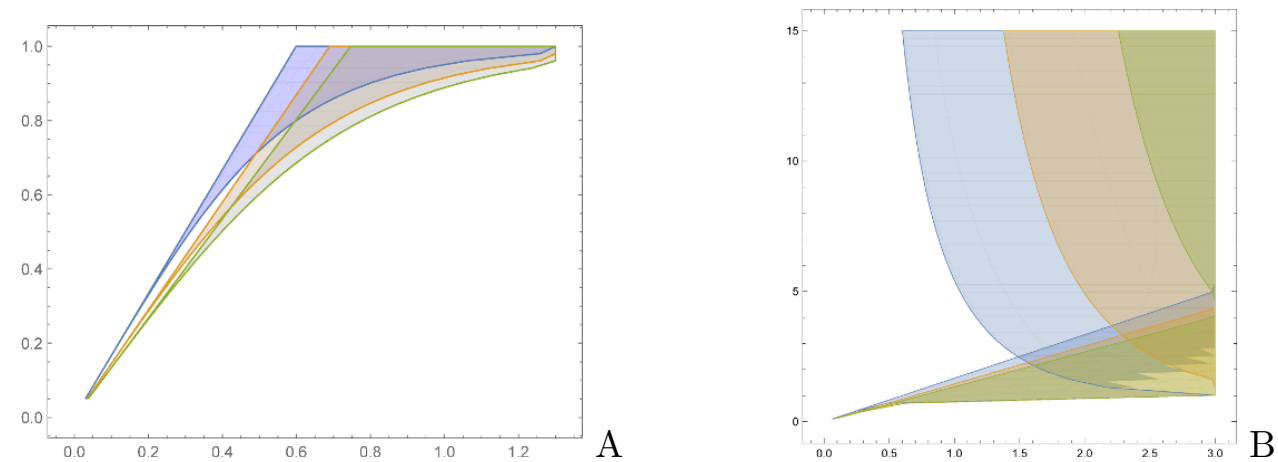

Figure 13. The same as in figure 12 for $\nu=2,3,4$ (blue,brown,green,respectively), $A$ : $z_{*} \leq 1, B$ : $z_{*}>1$

leads to a solution for the constants $\lambda, \mu, q$ and $\Lambda$ and the component $T_{00}^{\mathrm{s}}$ of the shell energy-momentum tensor. Thus, one finds that the ansatz (A.8) for the fields is valid. For $\nu=4$, the solution is formed by the values (2.15) for the constants $\lambda, \mu, q$ and $\Lambda$, as well as by the following expression of the shell energy-momentum:

$$
T_{00}^{\mathrm{S}}(v, r)=3 e^{-6 r} \frac{d m}{d v}
$$

\section{B Details on solutions to profiles equations}

\section{B.1 Equations (4.37), (4.38)}

In figure 12 and figure 13 we show the position of the singular point ( $x$-axis) of the solution with given $z_{*}(y$-axis $)$ and varying $v_{*}$. From figure 12 we see that for the given value of $z_{*} \leq 1$ varying $v_{*}$, say from $v_{*}=v_{1}$ to $v_{*}=v_{2}$, we get different positions of $l_{s}$ lying between $l_{s}\left(v_{1}\right)$ and $l_{s}\left(v_{2}\right)$. It is interesting to note that for small $z_{*}$ the position of the singular point is not considerably depends on value of $v_{*}$. For $z_{*} \rightarrow 1$ this dependence is more significant. We also see that one given value of $l_{s}$ corresponds to different values of $z_{*}$ and $v_{*}$. 

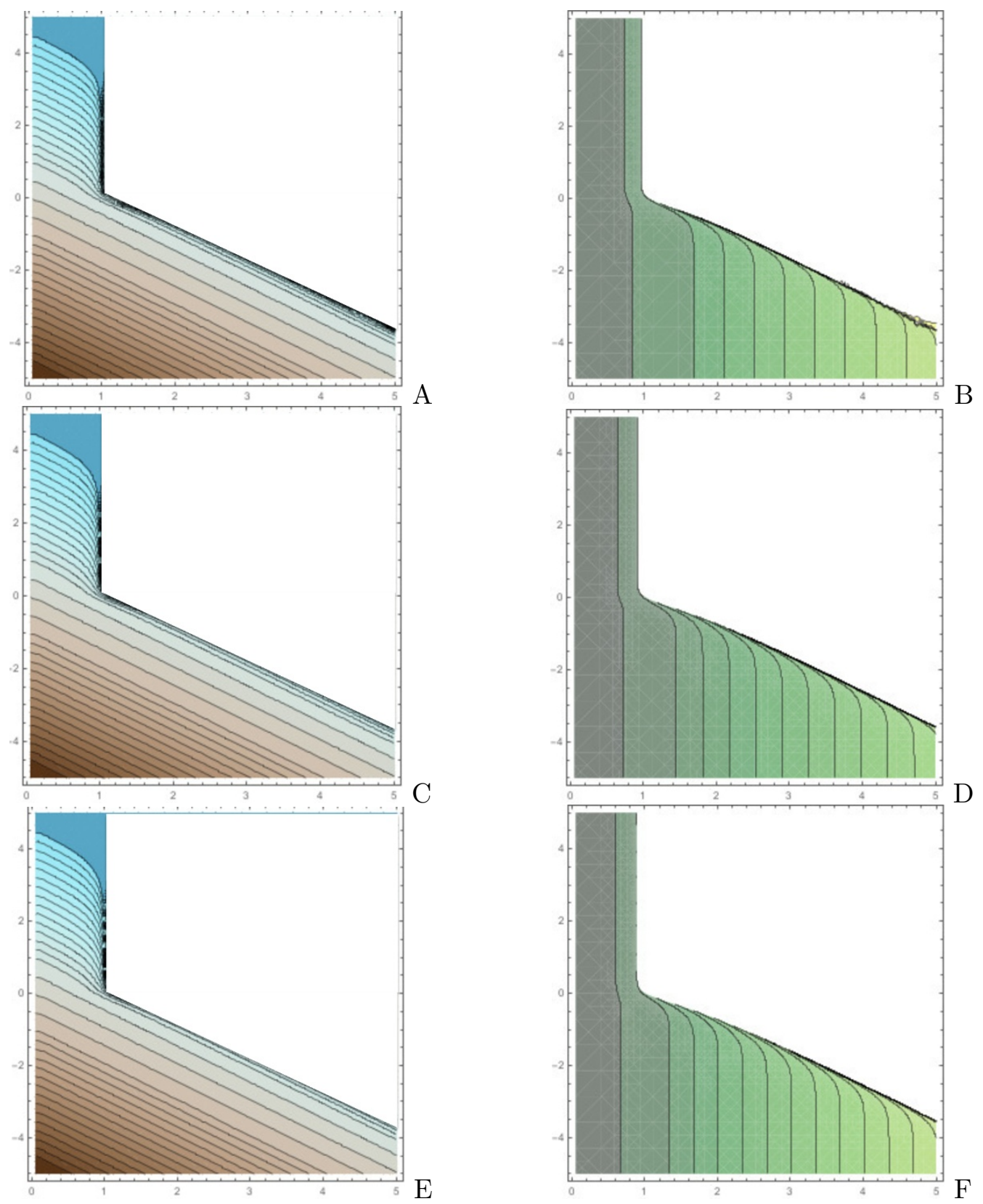

Figure 14. Left panel: contour plots for the boundary time as a function of initial conditions $z_{*}$ and $v_{*}$ for eqs. (4.37)-(4.38) for $\nu=2,3,4$ (A,C,E, respectively). Right panel: contour plots for $z\left(l_{\text {sing }}\right)$ as a function of $z_{*}$ and $v_{*}$ for eqs. (4.37)-(4.38) for $\nu=2,3,4(B, D, F$, respectively). The regions of white colour correspond to irrelevant initial conditions.

The position of the singular point for different values of critical exponent $\nu$ is presented in figure 13.

In figure 14 we present the contour plots for the boundary time and $z\left(l_{\text {sing }}\right)$ as functions of $z_{*}$ and $v_{*}$. The values of the initial conditions taken from regions of white colour yields solutions to eqs. (4.37)-(4.38), which do not obey the boundary constraints. 

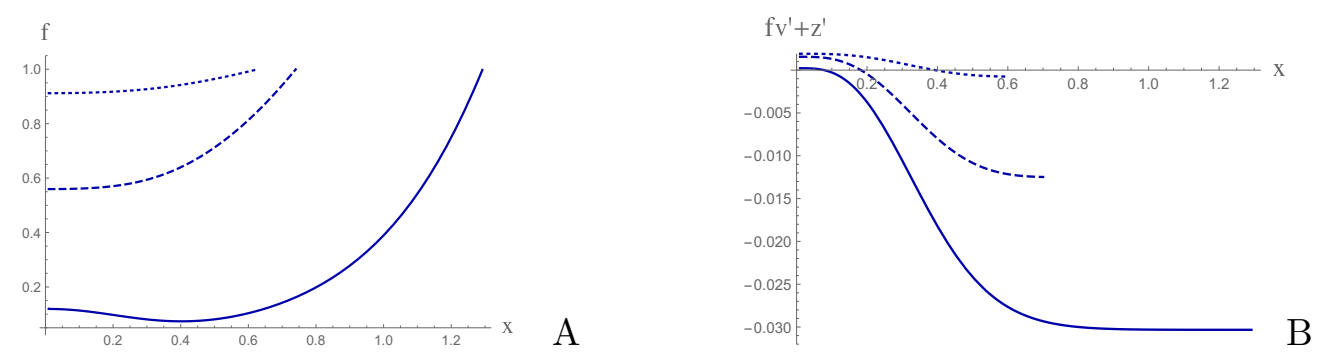

Figure 15. $A$ : the dependence of $f(x)$ on $x$ on the solutions to eqs. (4.37)-(4.38). B: the dependence of the quantity $f v^{\prime}+z^{\prime}$ on the solutions $z(x), v(x)$ to equations (4.37)-(4.38). For both plots $\nu=2$ and different masses: $m=1, m=0.5$ and $m=0.1$ shown by solid, dashed and dotted lines, respectively.

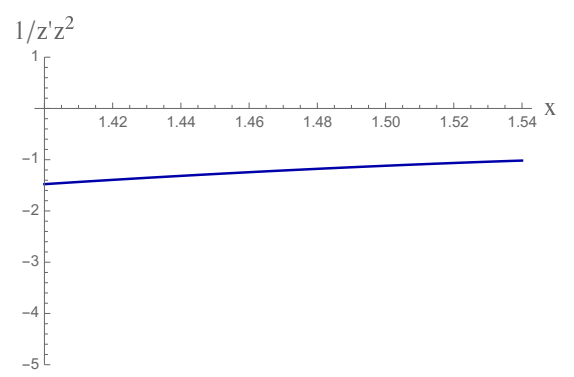

A

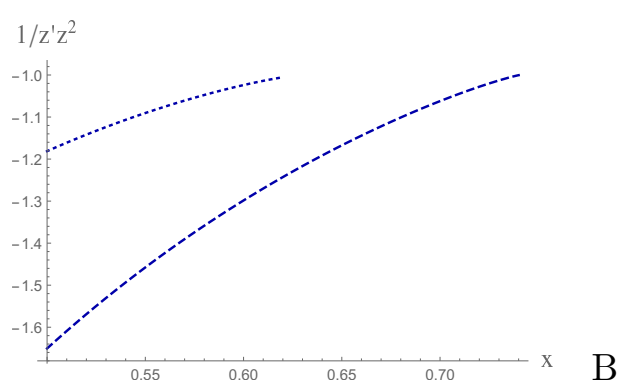

Figure 16. $A$ : the dependence of the quantity (4.46) on the solution $z(x)$ to equations (4.37)(4.38) for $\nu=2$ and $m=1$. B: the dependence of the quantity (4.46) on the solution $z(x)$ to equations (4.37)-(4.38) for different masses: $m=0.5$ and $m=0.1$ shown by dashed and dotted lines, respectively, $\nu=2$. In both cases $z_{*}=1$ and $\ell=0.63, \ell=0.75$ for $B$ and $C$, respectively.

It is also interesting to find the behaviour of the function $f(2.27)$ as a function of position on the constructed solutions to eqs. (4.37)-(4.38). In figure 15.A we present the behavior of $f(x)$ near to 1 in the region of the singular point.

In figure 16 we check the asymptotic behaviour of $\mathfrak{b}(z)$ defined by (4.46) on the solution $z(x)$ to equations (4.37)-(4.38) for $\nu=2$. For these solutions $z_{*}$ is taken to be 1 . We see that for $x \rightarrow \ell$, i.e. near the end of the profile, $\mathfrak{b}(z) \rightarrow 1$.

\section{B.2 Equations (4.63), (4.65)}

In figure 17 we show the profiles of the solutions to equations (4.63)-(4.65) for $z_{*}=1$ and different values of $\alpha$ and different $v_{*}$. We see that the profile for $\alpha=0.05$ is sharper, as can be obviously expected.

As it has been mentioned in section 4.2.2 on the region, where we can guarantee, that the quantity

$$
\mathcal{Q}=\frac{f(z) \dot{v}_{y}+\dot{z}_{y}}{2 z^{2-2 / \nu}}
$$

is conserved on the solution, we can say the solution can be approximated by the static solution in this region. We also present the dependence of the "quasi" conserved quantity $\mathcal{Q}$ on $x$ for $\nu=2$ in figure 19 .

In figure 20 we check the asymptotic behaviour of $\mathfrak{b}(z)$ defined by (4.72). 

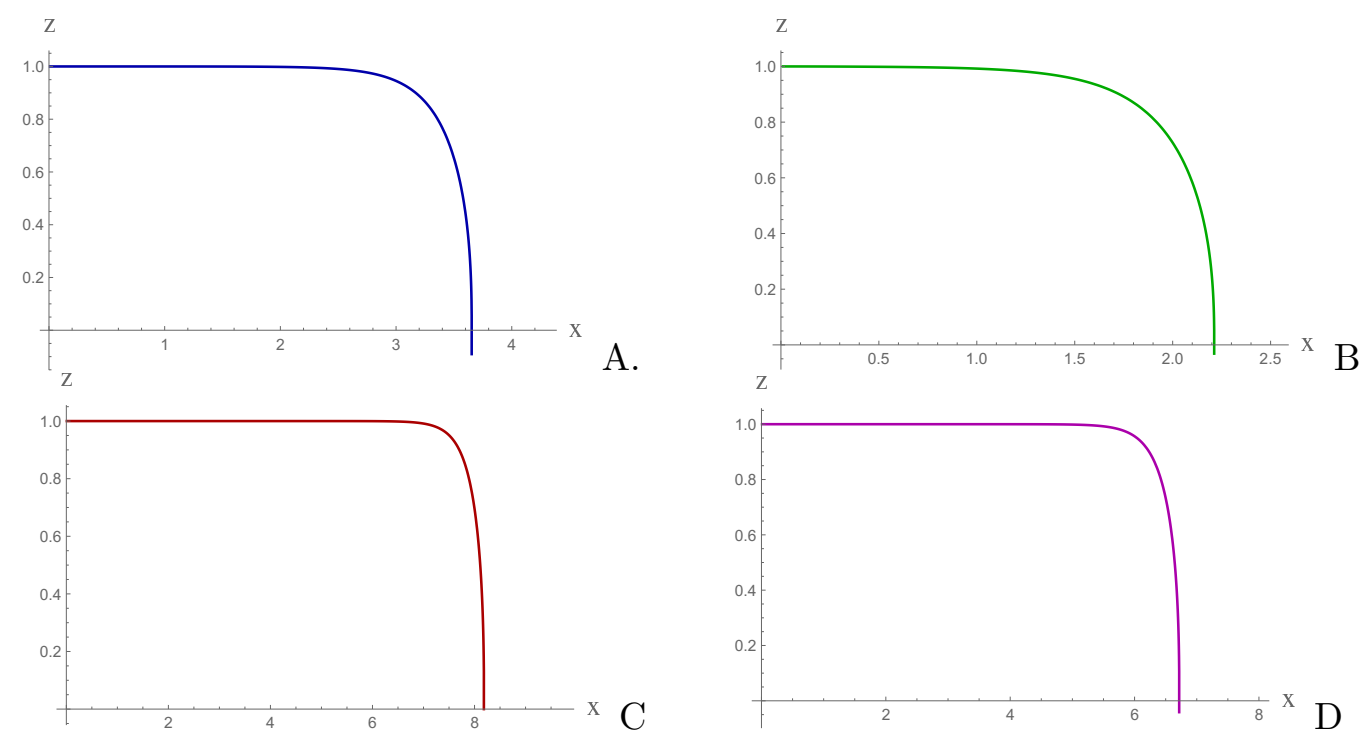

Figure 17. Forms of the profiles of the solutions to eqs. (4.63)-(4.65). A: $v_{*}=1, B: v_{*}=0.5$. For both cases $z_{*}=1$ and $\alpha=0.2$. $C: v_{*}=1, D: v_{*}=0.5$. In both cases $z_{*}=1$ and $\alpha=0.05$.


Figure 18. The dependence $f=f(x)$ on the solutions to eqs. (4.63)-(4.65). $A: v_{*}=1, B$ : $v_{*}=0.5$. For both cases $z_{*}=1$.
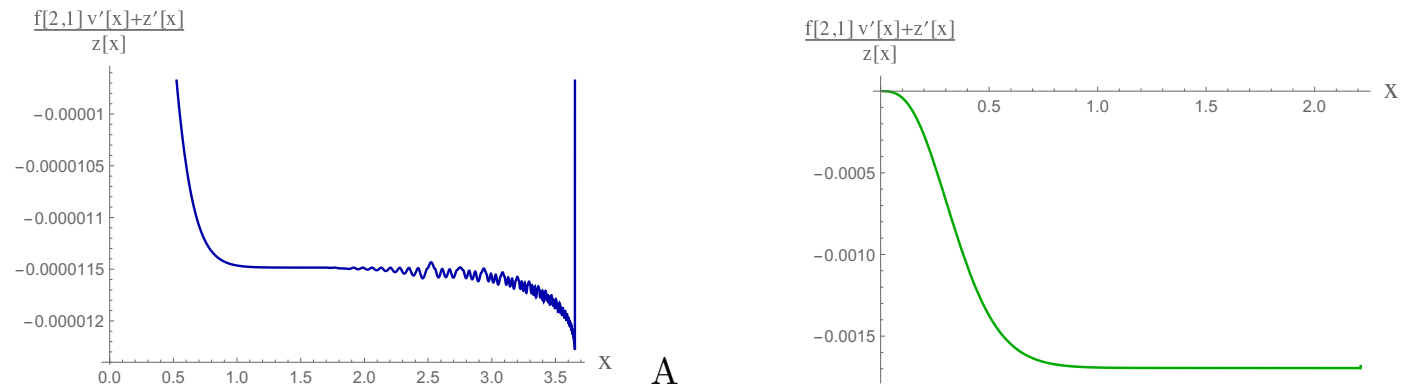

$\mathrm{B}$

Figure 19. Behaviour of a "quasi" conserved quantity $\mathcal{Q}$ for $\nu=2$. $A: v_{*}=1, B: v_{*}=0.5$. For both cases $z_{*}=1$ 



A

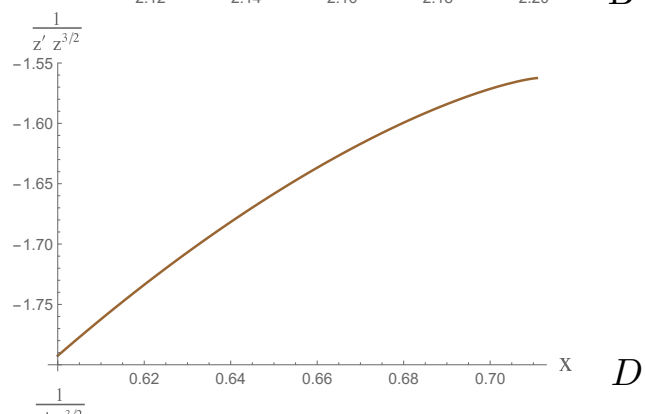

$\mathrm{E}$

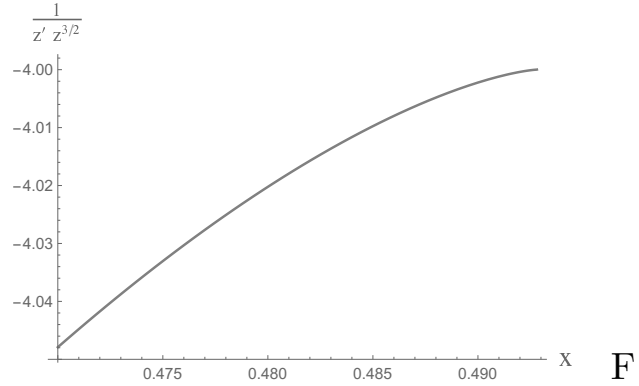

Figure 20. Check of asymptotic behaviour of $\mathfrak{b}(z)$ near $z=0 . A: v_{*}=1, B: v_{*}=0.5$. In both cases $z_{*}=1 C: v_{*}=1, D: v_{*}=0.1$. In both cases $z_{*}=0.8 ; E: v_{*}=1, F: v_{*}=0.1$. In both cases $z_{*}=0.5$ The asymptotic is $1 / z_{*}^{2}$, i.e. for $z_{*}=0.8$ it is 1.5625 and for $z_{*}=0.5$ it is 4 .
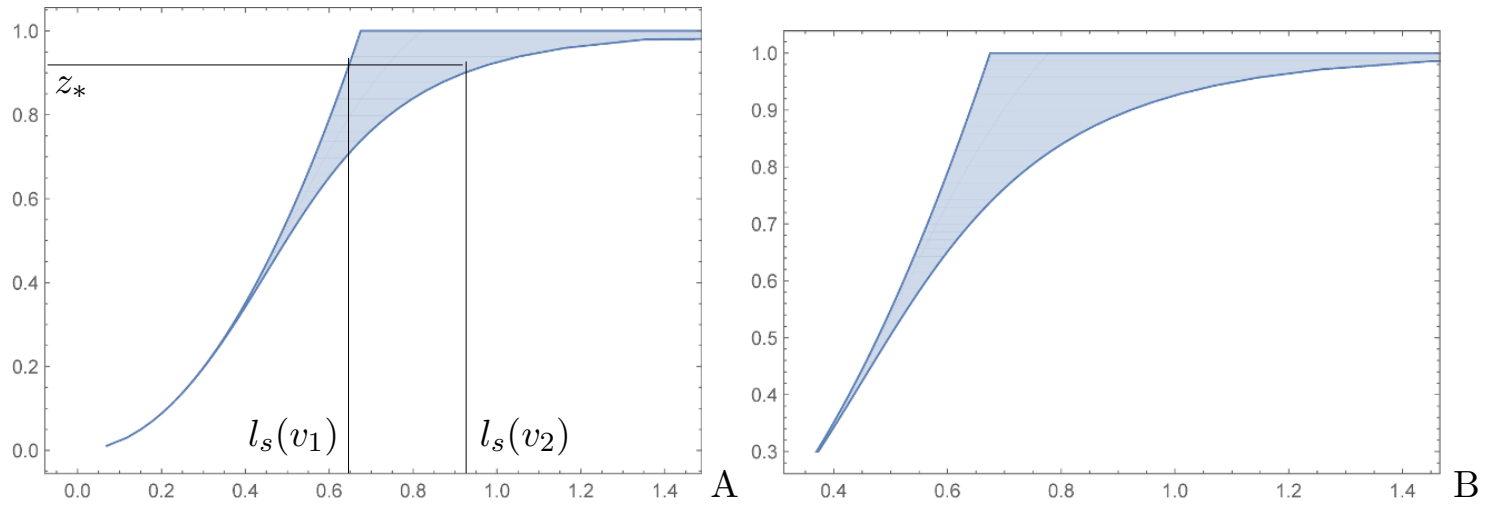

Figure 21. A: positions of the singular point for given $z_{*} \leq 1$ and given $v_{*}$ belonging to the variety $v_{1} \leq v_{*} \leq v_{2}, l_{s}\left(v_{1}\right) \leq l_{s}\left(v_{1}\right) \leq l_{s}\left(v_{2}\right)$. In this plot $v_{1}=-5, v_{2}=25 . \nu=2 . B$ : the same for $\alpha=0.05$. 

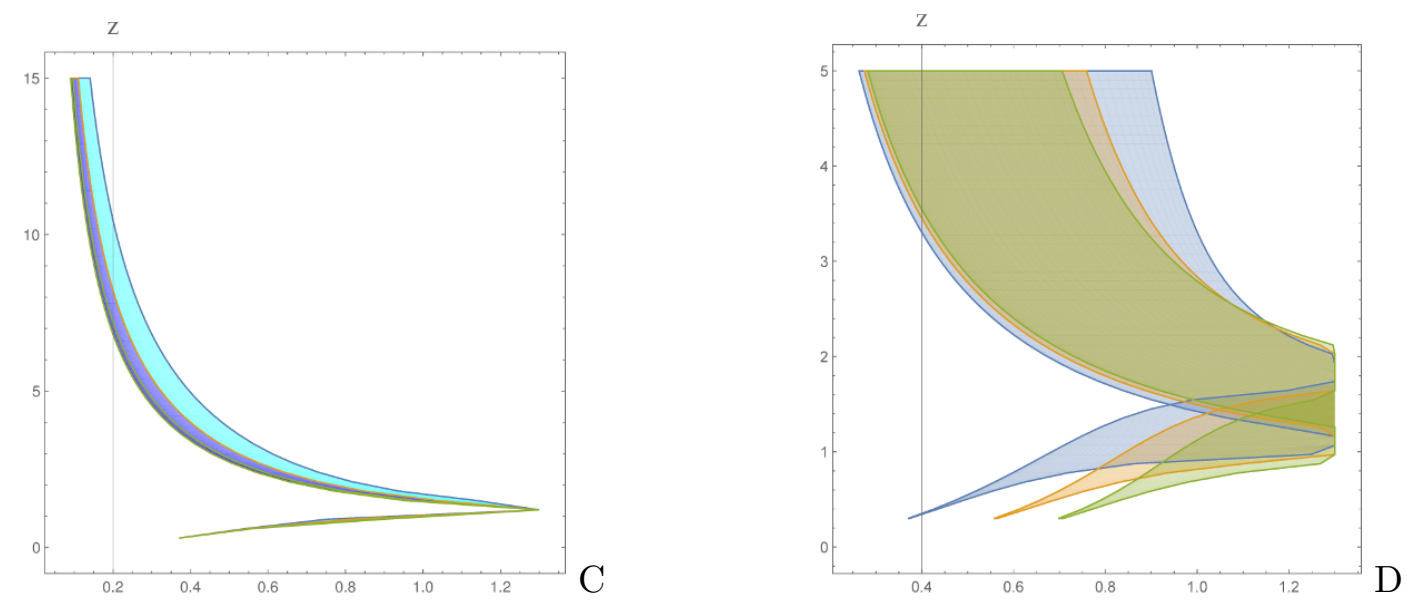

Figure 22. $A$ : positions of the singular point for given $z_{*}, 0<z_{*}<15$ and given $v_{*}$ belonging to the variety $v_{1} \leq v_{*} \leq v_{2}, v_{1}=-5, v_{2}=25 . \nu=2$. B: the same for $0<z_{*}<5$ and $\nu=2,3,4$.

We present the dependence of $l_{\text {sing }}$ on the turning point $z_{*}$ and $v_{*}$ in figure 21 and figure 22. Here we again observe that one can get different positions of $l_{s}$ in the range from $l_{s}\left(v_{1}\right)$ to $l_{s}\left(v_{2}\right)$ varying $v_{*}$ and fixing $z_{*}$.

In the three left panels of figure 23 we show contour plots for the boundary time depending on the initial conditions $z_{*}, v_{*}$ for $\nu=2,3,4$. In the three right panels of figure 23 we present contour plots for $z\left(l_{\text {sing }}\right)$ as a function of initial conditions $z_{*}, v_{*}$ for $\nu=2,3,4$. As in the previous case, regions of white colour correspond to the irrelevant initial conditions. 



Figure 23. Left panel: contour plots for the boundary time as a function of initial conditions $z_{*}$ and $v_{*}$ for eqs. (4.63)-(4.65) for $\nu=2,3,4$ (A,C,E, respectively). Right panel: contour plots for $z\left(l_{\text {sing }}\right)$ as a function of $z_{*}$ and $v_{*}$ for eqs. (4.63)-(4.65) for $\nu=2,3,4(B, D, F$, respectively). The regions of white colour correspond to irrelevant initial conditions.

Open Access. This article is distributed under the terms of the Creative Commons Attribution License (CC-BY 4.0), which permits any use, distribution and reproduction in any medium, provided the original author(s) and source are credited. 


\section{References}

[1] J. Casalderrey-Solana, H. Liu, D. Mateos, K. Rajagopal and U.A. Wiedemann, Gauge/string duality, hot QCD and heavy ion collisions, arXiv:1101.0618 [INSPIRE].

[2] I.Ya. Aref'eva, Holographic approach to quark-gluon plasma in heavy ion collisions, Phys. Usp. 57 (2014) 527.

[3] O. DeWolfe, S.S. Gubser, C. Rosen and D. Teaney, Heavy ions and string theory, Prog. Part. Nucl. Phys. 75 (2014) 86 [arXiv: 1304.7794] [INSPIRE].

[4] M. Strickland, Thermalization and isotropization in heavy-ion collisions, Pramana 84 (2015) 671 [arXiv: 1312.2285] [INSPIRE].

[5] D. Giataganas, Probing strongly coupled anisotropic plasma, JHEP 07 (2012) 031 [arXiv:1202.4436] [INSPIRE].

[6] D. Giataganas, Observables in strongly coupled anisotropic theories, PoS (Corfu2012) 122 [arXiv: 1306.1404] [INSPIRE].

[7] S.S. Gubser, S.S. Pufu and A. Yarom, Entropy production in collisions of gravitational shock waves and of heavy ions, Phys. Rev. D 78 (2008) 066014 [arXiv:0805.1551] [INSPIRE].

[8] J.L. Albacete, Y.V. Kovchegov and A. Taliotis, Modeling heavy ion collisions in AdS/CFT, JHEP 07 (2008) 100 [arXiv:0805.2927] [INSPIRE].

[9] L. Álvarez-Gaumé, C. Gomez, A. Sabio Vera, A. Tavanfar and M.A. Vazquez-Mozo, Critical formation of trapped surfaces in the collision of gravitational shock waves, JHEP 02 (2009) 009 [arXiv:0811.3969] [INSPIRE].

[10] P.M. Chesler and L.G. Yaffe, Horizon formation and far-from-equilibrium isotropization in supersymmetric Yang-Mills plasma, Phys. Rev. Lett. 102 (2009) 211601 [arXiv:0812.2053] [INSPIRE].

[11] S. Lin and E. Shuryak, Grazing collisions of gravitational shock waves and entropy production in heavy ion collision, Phys. Rev. D 79 (2009) 124015 [arXiv:0902.1508] [INSPIRE].

[12] I. Ya. Aref'eva, A.A. Bagrov and E.A. Guseva, Critical formation of trapped surfaces in the collision of non-expanding gravitational shock waves in de Sitter space-time, JHEP 12 (2009) 009 [arXiv: 0905.1087] [INSPIRE].

[13] P.M. Chesler and L.G. Yaffe, Holography and colliding gravitational shock waves in asymptotically $A d S_{5}$ spacetime, Phys. Rev. Lett. 106 (2011) 021601 [arXiv:1011.3562] [INSPIRE].

[14] I.Y. Arefeva, A.A. Bagrov and E.O. Pozdeeva, Holographic phase diagram of quark-gluon plasma formed in heavy-ions collisions, JHEP 05 (2012) 117 [arXiv:1201.6542] [INSPIRE].

[15] E. Kiritsis and A. Taliotis, Multiplicities from black-hole formation in heavy-ion collisions, JHEP 04 (2012) 065 [arXiv:1111.1931] [INSPIRE].

[16] I. Ya. Aref'eva, E.O. Pozdeeva and T.O. Pozdeeva, Holographic estimation of multiplicity and membranes collision in modified spaces $A d S_{5}$, Theor. Math. Phys. 176 (2013) 861 [arXiv:1401.1180] [INSPIRE].

[17] V. Balasubramanian et al., Holographic thermalization, Phys. Rev. D 84 (2011) 026010 [arXiv:1103.2683] [INSPIRE]. 
[18] J. Abajo-Arrastia, J. Aparicio and E. Lopez, Holographic evolution of entanglement entropy, JHEP 11 (2010) 149 [arXiv: 1006.4090] [INSPIRE].

[19] R. Callan, J.-Y. He and M. Headrick, Strong subadditivity and the covariant holographic entanglement entropy formula, JHEP 06 (2012) 081 [arXiv: 1204.2309] [INSPIRE].

[20] E. Caceres and A. Kundu, Holographic thermalization with chemical potential, JHEP 09 (2012) 055 [arXiv: 1205.2354] [INSPIRE].

[21] I. Ya. Arefeva and I.V. Volovich, On holographic thermalization and dethermalization of quark-gluon plasma, arXiv:1211.6041 [INSPIRE].

[22] M. Taylor, Non-relativistic holography, arXiv:0812.0530 [INSPIRE].

[23] M. Taylor, Lifshitz holography, Class. Quant. Grav. 33 (2016) 033001 [arXiv:1512.03554] [INSPIRE].

[24] I. Ya. Aref'eva and A.A. Golubtsova, Shock waves in Lifshitz-like spacetimes, JHEP 04 (2015) 011 [arXiv:1410.4595] [INSPIRE].

[25] T. Azeyanagi, W. Li and T. Takayanagi, On string theory duals of Lifshitz-like fixed points, JHEP 06 (2009) 084 [arXiv:0905.0688] [INSPIRE].

[26] D. Mateos and D. Trancanelli, Thermodynamics and instabilities of a strongly coupled anisotropic plasma, JHEP 07 (2011) 054 [arXiv: 1106.1637] [INSPIRE].

[27] D.S. Ageev, I. Ya. Aref'eva, A.A. Golubtsova and E. Gourgoulhon, Holographic Wilson loops in Lifshitz-like backgrounds, arXiv:1606.03995 [INSPIRE].

[28] SageManifolds webpage, http://sagemanifolds.obspm.fr/.

[29] SageMath webpage, http://www.sagemath.org/.

[30] S. Chakrabortty, S. Chakraborty and N. Haque, Brownian motion in strongly coupled, anisotropic Yang-Mills plasma: a holographic approach, Phys. Rev. D 89 (2014) 066013 [arXiv: 1311.5023] [INSPIRE].

[31] D. Giataganas and H. Soltanpanahi, Heavy quark diffusion in strongly coupled anisotropic plasmas, JHEP 06 (2014) 047 [arXiv:1312.7474] [INSPIRE].

[32] L. Cheng, X.-H. Ge and S.-J. Sin, Anisotropic plasma with a chemical potential and scheme-independent instabilities, Phys. Lett. B 734 (2014) 116 [arXiv:1404.1994] [INSPIRE].

[33] V. Keranen, E. Keski-Vakkuri and L. Thorlacius, Thermalization and entanglement following a non-relativistic holographic quench, Phys. Rev. D 85 (2012) 026005 [arXiv:1110.5035] [INSPIRE].

[34] I. Ya. Aref'eva, Formation time of quark-gluon plasma in heavy-ion collisions in the holographic shock wave model, Theor. Math. Phys. 184 (2015) 1239 [Teor. Mat. Fiz. 184 (2015) 398] [arXiv: 1503.02185] [INSPIRE].

[35] M. Alishahiha, A.F. Astaneh and M.R. Mohammadi Mozaffar, Thermalization in backgrounds with hyperscaling violating factor, Phys. Rev. D 90 (2014) 046004 [arXiv: 1401.2807] [INSPIRE].

[36] P. Fonda, L. Franti, V. Keränen, E. Keski-Vakkuri, L. Thorlacius and E. Tonni, Holographic thermalization with Lifshitz scaling and hyperscaling violation, JHEP 08 (2014) 051 [arXiv: 1401.6088] [INSPIRE]. 
[37] V. Keranen and L. Thorlacius, Thermal correlators in holographic models with Lifshitz scaling, Class. Quant. Grav. 29 (2012) 194009 [arXiv:1204.0360] [INSPIRE].

[38] V.E. Hubeny, Extremal surfaces as bulk probes in AdS/CFT, JHEP 07 (2012) 093 [arXiv: 1203.1044] [INSPIRE].

[39] H. Liu and S.J. Suh, Entanglement growth during thermalization in holographic systems, Phys. Rev. D 89 (2014) 066012 [arXiv:1311.1200] [InSPIRE].

[40] U. Kol, C. Núñez, D. Schofield, J. Sonnenschein and M. Warschawski, Confinement, phase transitions and non-locality in the entanglement entropy, JHEP 06 (2014) 005 [arXiv: 1403.2721] [INSPIRE].

[41] S. Fischetti and D. Marolf, Complex entangling surfaces for AdS and Lifshitz black holes?, Class. Quant. Grav. 31 (2014) 214005 [arXiv:1407.2900] [INSPIRE].

[42] P. Fonda, D. Seminara and E. Tonni, On shape dependence of holographic entanglement entropy in $A d S_{4} / C F T_{3}, J H E P 12$ (2015) 037 [arXiv:1510.03664] [INSPIRE].

[43] R. Kallosh and A.W. Peet, Dilaton black holes near the horizon, Phys. Rev. D 46 (1992) R5223 [hep-th/9209116] [inSPIRE].

[44] A. Donos, J.P. Gauntlett and C. Pantelidou, Spatially modulated instabilities of magnetic black branes, JHEP 01 (2012) 061 [arXiv:1109.0471] [INSPIRE].

[45] A. Donos, J.P. Gauntlett and C. Pantelidou, Magnetic and electric AdS solutions in stringand M-theory, Class. Quant. Grav. 29 (2012) 194006 [arXiv:1112.4195] [InSPIRE].

[46] E. D'Hoker and P. Kraus, Charge expulsion from black brane horizons and holographic quantum criticality in the plane, JHEP 09 (2012) 105 [arXiv:1202.2085] [INSPIRE].

[47] P. Vaidya, The external field of a radiating star in general relativity, Curr. Sci. 12 (1943) 183.

[48] S. Ryu and T. Takayanagi, Holographic derivation of entanglement entropy from AdS/CFT, Phys. Rev. Lett. 96 (2006) 181602 [hep-th/0603001] [INSPIRE].

[49] S. Ryu and T. Takayanagi, Aspects of holographic entanglement entropy, JHEP 08 (2006) 045 [hep-th/0605073] [inSPIRE].

[50] V.E. Hubeny, M. Rangamani and T. Takayanagi, A covariant holographic entanglement entropy proposal, JHEP 07 (2007) 062 [arXiv:0705.0016] [INSPIRE].

[51] T. Albash and C.V. Johnson, Evolution of holographic entanglement entropy after thermal and electromagnetic quenches, New J. Phys. 13 (2011) 045017 [arXiv: 1008.3027] [INSPIRE].

[52] A. Allais and E. Tonni, Holographic evolution of the mutual information, JHEP 01 (2012) 102 [arXiv: 1110.1607 ] [INSPIRE].

[53] E. Caceres, M. Sanchez and J. Virrueta, Holographic entanglement entropy in time dependent Gauss-Bonnet gravity, arXiv:1512.05666 [INSPIRE].

[54] K. Goldstein, S. Kachru, S. Prakash and S.P. Trivedi, Holography of charged dilaton black holes, JHEP 08 (2010) 078 [arXiv:0911.3586] [INSPIRE].

[55] C. Charmousis, B. Gouteraux, B.S. Kim, E. Kiritsis and R. Meyer, Effective holographic theories for low-temperature condensed matter systems, JHEP 11 (2010) 151 [arXiv: 1005.4690] [INSPIRE].

[56] N. Iizuka, N. Kundu, P. Narayan and S.P. Trivedi, Holographic Fermi and non-Fermi liquids with transitions in dilaton gravity, JHEP 01 (2012) 094 [arXiv: 1105.1162] [INSPIRE]. 